\title{
Analytical Calculation of Four-Point Correlations for a Simple Model of Cages Involving Numerous Particles
}

\author{
Ooshida Takesh:* \\ Department of Mechanical and Aerospace Engineering, Tottori University, Tottori 680-8552, Japan \\ Susumu Goto \\ Graduate School of Engineering Science, Osaka University, Toyonaka, Osaka 560-8531, Japan \\ Takeshi Matsumoto \\ Division of Physics and Astronomy, Graduate School of Science, Kyoto University, Kyoto 606-8502, Japan \\ Akio Nakahara \\ Laboratory of Physics, College of Science and Technology, \\ Nihon University, Funabashi, Chiba 274-8501, Japan \\ Michio Otsuk $\oplus$ \\ Department of Physics and Mathematics, Aoyama Gakuin University, Sagamihara, Kanagawa 229-8558, Japan
}

(Dated: August 18, 2018)

\begin{abstract}
Dynamics of a one-dimensional system of Brownian particles with short-range repulsive interaction (diameter $\sigma$ ) is studied with a liquid-theoretical approach. The mean square displacement, the two-particle displacement correlation, and the overlap-density-based generalized susceptibility are calculated analytically by way of the Lagrangian correlation of the interparticulate space, instead of the Eulerian correlation of density that is commonly used in the standard mode-coupling theory. In regard to the mean square displacement, the linear analysis reproduces the established result on the asymptotic subdiffusive behavior of the system. A finite-time correction is given by incorporating the effect of entropic nonlinearity with a Lagrangian version of mode-coupling theory. The notorious difficulty in derivation of the mode-coupling theory concerning violation of the fluctuation-dissipation theorem is found to disappear by virtue of the Lagrangian description. The Lagrangian description also facilitates analytical calculation of four-point correlations in the space-time, such as the twoparticle displacement correlation. The two-particle displacement correlation, which is asymptotically self-similar in the space-time, illustrates how the cage effect confines each particle within a short radius on one hand and creates collective motion of numerous particles on the other hand. As the time elapses, the correlation length grows unlimitedly, and the generalized susceptibility based on the overlap density converges to a finite value which is an increasing function of the density. The distribution function behind these dynamical four-point correlations and its extension to threedimensional cases, respecting the tensorial character of the two-particle displacement correlation, are also discussed.
\end{abstract}

\section{INTRODUCTION}

Confined dynamics of Brownian particles has been studied for many reasons, such as its relevance to microfluidic devices [1, 2], molecular biology [3, 4], and energetics of micromachines [5, 6]. Most notably, the problems are intriguing because the confinement makes even the simplest cases non-trivial, not to speak of more challenging cases in which the particle-particle interaction comes into play. The simplest and apparently easier cases are exemplified by diffusion of non-interacting Brownian particles in a cylindrical pore with a varying cross section [7]. The diffusive dynamics is then described by a spatially one-dimensional Fokker-Planck equation for particles in a rugged free-energy landscape [8]. Due to the entropic

\footnotetext{
*E-mail: ooshida@damp.tottori-u.ac.jp

${ }^{\dagger}$ Present address: Department of Materials Science, Shimane University, Matsue 690-8504, Japan
}

nature of this free-energy landscape, the diffusion under an external driving force exhibits a peculiar temperature dependence [9]; it is also sensitive to the particle size, which can be applied to a design of a device for sorting particles [10].

Interaction among the particles makes the problem of confined dynamics a real challenge. It means that the confinement is caused by the particles themselves and the motion is thus slowed down, as if each particle is constrained in a cage that consists of its neighbors. This kind of mutual hindrance of motion, which has been studied in connection with the glass transition [11] and now in a broader context [12], is known by the name of the cage effect.

To see how the slowdown of the dynamics due to the cage effect is studied quantitatively, let us consider a dense colloidal suspension modeled as a system of interacting Brownian particles, denoting the position vector of the $j$-th particle with $\mathbf{r}_{j}(t)$. The slow dynamics is 
studied by defining the (particle-scale) density field as

$$
\rho(\mathbf{r}, t)=\sum_{j} \delta\left(\mathbf{r}-\mathbf{r}_{j}(t)\right)=\rho_{0}+\sum_{\mathbf{k}} \hat{\rho}(\mathbf{k}, t) e^{-i \mathbf{k} \cdot \mathbf{r}}
$$

and focusing on its correlations, such as the intermediate scattering function (the dynamical structure factor), $F(k, t) \propto\langle\hat{\rho}(\mathbf{k}, t) \hat{\rho}(-\mathbf{k}, 0)\rangle$, with $\hat{\rho}$ denoting the Fourier component of the density field. As the mean density $\rho_{0}$ increases or the temperature $T$ decreases, the cages have stronger effect, which results in the extremely slow relaxation of $F(k, t)$. This behavior of $F(k, t)$ has been reproduced theoretically, at least to some extent, by the mode-coupling theory (MCT) $13-15]$, which consists in the derivation of an equation for $F(k, t)$ in the form of an integro-differential equation,

$$
\left(\partial_{t}+D_{\mathrm{c}} k^{2}\right) F(k, t)=-\int_{0}^{t} \mathrm{~d} t^{\prime} M\left(k, t-t^{\prime}\right) \partial_{t^{\prime}} F\left(k, t^{\prime}\right),
$$

with $D_{\text {c }}$ denoting the collective diffusion constant; the cage effect is incorporated via the memory kernel $M$ which is a quadratic functional of $F$. In spite of this success, however, MCT suffers from several difficulties and has its own limitations [11, 15]. Since theoretical understanding of glassy dynamics still remains far from being resolved, a methodological insight into kinetic approaches to glassy systems, which will permit an improvement over the existing theories such as MCT, is highly desired.

It is one aspect of the cage effect that each particle is confined within a short radius, while it has another aspect that concerns long length scales. The slowdown of the glassy dynamics is now regarded as attributable to dynamical heterogeneity [16, 17], which refers to the presence of collective motion with some lifetime and correlation length. Unfortunately, kinetic-theoretical treatment of this collective motion is formidably difficult, as its correct description requires a four-point space-time correlation, such as $\chi_{4}$ that will be explained later [see Eq. (5.8) in Sec. $\mathrm{V}$. As far as we know, analytical calculation of four-point correlations has been infeasible except for some special cases such as linear elastic bodies and kinetically constrained models on a lattice [18]. In regard to MCT, we must emphasize here that MCT targets on the dynamical structure factor $F(k, t)$ and not on four-point correlations. As long as the standard variables such as $\hat{\rho}(\mathbf{k}, t)$ are used, a four-point correlation function implies a four-body correlation. Since MCT is a closure theory in which quadruple (four-body) correlations are approximated by products of $F$, it is unlikely to describe four-point correlations accurately. Although a calculation of three-point correlation within the MCT approximation was recently reported [19], still $\chi_{4}$ remains insurmountable.

A breakthrough may be found, through a profound study of a simpler system, by developing a method that can describe the two aspects of the cage effect simultaneously, namely the short-ranged particle interaction and the long-ranged dynamical correlation. To make progress in this direction, here we develop a nonlinear theory for

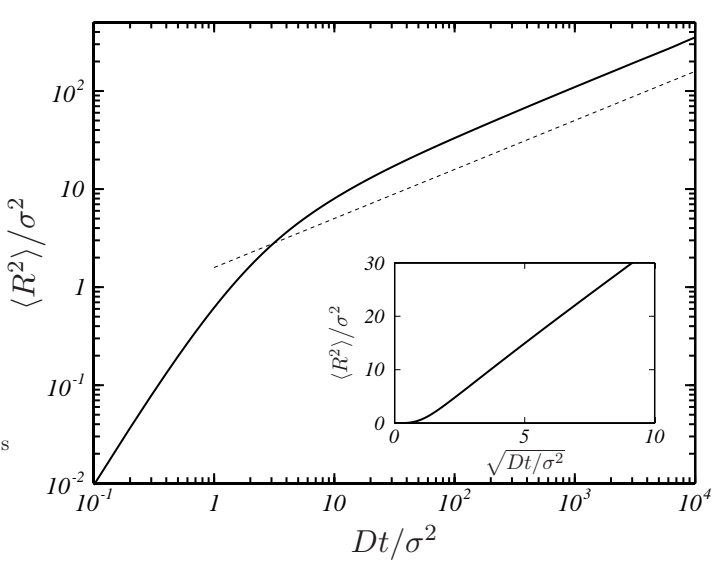

FIG. 1: The mean square displacement $\left\langle R^{2}\right\rangle$ in SFD, plotted as a function of $t$. The dotted line indicates the slope for $t^{1 / 2}$. The system size is specified as $N=2^{15}=32768$ and $\rho_{0}=N / L=0.25 \sigma^{-1}$. In the inset, the same $\left\langle R^{2}\right\rangle$ is plotted against $\sqrt{t}$ for $0<\sqrt{D t}<10 \sigma$.

a one-dimensional system of interacting Brownian particles:

$$
m \ddot{X}_{i}=-\mu \dot{X}_{i}-\frac{\partial}{\partial X_{i}} \sum_{j<k} V\left(X_{k}-X_{j}\right)+\mu f_{i}(t),
$$

whose behavior is known by the name of single-file diffusion (SFD) 20 31], and which has been studied also as a model of glassy dynamics with ideal cages 32,34$]$. In the Langevin equation (1.2), $X_{i}$ represents the position of the $i$-th particle, and the meaning of the other symbols should be self-evident. Without the interaction $(V=0)$, the statistically averaged or coarse-grained density field would be governed by the simple diffusion equation, with the diffusion constant $D=k_{\mathrm{B}} T / \mu$. Here we adopt for $V$ a short-ranged repulsive potential, such as Eq. (A1) in Appendix $\mathrm{A}$ so that the system exhibits a liquid-like structure factor [35]. The statistics of the random forcing, $\mu f_{i}(t)$, are given by Eq. (A2).

As will be clarified below, we propose to contribute two things to the theory of SFD. Firstly, we establish a framework for systematic treatment of the nonlinear effect of density fluctuations in the form of an MCT-like equation, which gives a correction to the asymptotic theory. In other words, we demonstrate how to take into account the free-energy landscape of the system beyond the linear (harmonic) approximation [36]. Secondly, we calculated some four-point space-time correlations in SFD, hoping that they will be useful as tools to quantify collective dynamics. These two concepts, namely MCT and four-point space-time correlations, are imported from the theory of glassy liquids, but in the original context of three-dimensional systems it has been difficult to combine them. In a simpler problem of SFD, we can demonstrate how they should be combined, so that the result will be exported back to the theory of glassy liquids in the near future.

Before clarifying the key idea for these tasks, we need to notice that the four-point correlation indicates col- 
lective motion associated with the slowness of SFD. By "slow" we mean that SFD is subdiffusive [23, 25, 26]: in regard to long-time behavior of the mean square displacement (MSD), denoted by $\left\langle R_{j}^{2}\right\rangle$ with

$$
R_{j}=R_{j}(t)=X_{j}(t)-X_{j}(0),
$$

it is known exactly that $\left\langle R_{j}^{2}\right\rangle$ for SFD behaves like $\sqrt{t}$ [see Eq. (1.4) shown later], which is slower than $\left\langle R_{j}^{2}\right\rangle \propto t$ expected for the normal diffusion. The subscript in $\left\langle R_{j}^{2}\right\rangle$ can be omitted as we assume that the system is statistically uniform. The subdiffusive law, $\left\langle R^{2}\right\rangle \propto \sqrt{t}$, is readily confirmed by simulation of a system with $N$ particles in a periodic box of the size $L$. In the calculations shown in Fig. 1, the mean density, $\rho_{0}=N / L$, equals $0.25 \sigma^{-1}$, and the system is statistically steady. For computational details, see Appendix A. The finite size effect is eliminated by taking sufficiently large $L$ and interpreting the word "long-time" as $\rho_{0}^{-2} \ll D t \ll L^{2} \rightarrow \infty$. For the long-time regime in this sense, the asymptotic MSD is given by

$$
\left\langle R^{2}\right\rangle=\frac{2 S}{\rho_{0}} \sqrt{\frac{D_{\mathrm{c}} t}{\pi}} \propto t^{1 / 2},
$$

where $S=S(0)$ denotes the long wave limiting value of the static structure factor $S(k)$. For particles with a welldefined diameter $\sigma$, an equation equivalent to Eq. (1.4) was derived by Hahn and Kärger [25]. Later, Kollmann [26] demonstrated that Eq. (1.4) holds for systems with arbitrary interaction potential, as long as the range of the interaction is finite. We note that Kollmann also needed to calculate a kind of four-point correlation $\left(\kappa^{(2)}\right.$ in his notation) in derivation of Eq. (1.4).

The slowness of SFD is ultimately due to the presence of the repulsive potential term in Eq. (1.2). Then there is a question: Does a straightforward application of MCT to Eq. (1.2) reproduce the subdiffusive law (1.4)? Unfortunately, the answer is negative. The MCT equation for the tagged particle, in any spatial dimension, reads as

$$
\left(\partial_{t}+D k^{2}\right) F_{\mathrm{S}}(k, t)=-\int_{0}^{t} \mathrm{~d} t^{\prime} M_{\mathrm{S}}\left(k, t-t^{\prime}\right) \partial_{t^{\prime}} F_{\mathrm{S}}\left(k, t^{\prime}\right),
$$

where

$$
F_{\mathrm{S}}(k, t)=\left\langle\hat{\rho}_{j}(\mathbf{k}, t) \hat{\rho}_{j}(-\mathbf{k}, 0)\right\rangle=\left\langle e^{-\mathbf{i k} \cdot \mathbf{R}_{j}}\right\rangle,
$$

with $\mathbf{R}_{j}=\mathbf{r}_{j}(t)-\mathbf{r}_{j}(0)$, is the self part of the intermediate scattering function $F$, and $M_{\mathrm{S}}$ is the memory kernel which is a bilinear functional of $F$ and $F_{\mathrm{S}}$. The MSD is given by expanding $F_{\mathrm{S}}$ in power series of $k$, as $F_{\mathrm{S}}=1-\frac{1}{2} k^{2}\left\langle R^{2}\right\rangle+\cdots$. The asymptotic behavior of MCT equations (1.1) and (1.5) with standard memory kernels, in any spatial dimension, is mostly the same as the schematic MCT equation, whose asymptotic solutions are either arrested or subject to an exponential decay [14]. This implies that the dynamical constraint by the long-lived cages in SFD is not described accurately enough by the standard MCT. Essentially the same difficulty occurs in MCT for rod polymers [37], as it predicts that the self-diffusion coefficient becomes isotropic for large aspect ratio and therefore fails to describe the entanglement effects. However, in this particular case 37], an alternative version of MCT-like kinetic theory can be developed. Using SFD as an illustrative example, Miyazaki [38, 39] suggested that a scheme for improved treatment of four-point correlation should be sought for.

The key idea of the present study for improved treatment of slow dynamics is adoption of the label variable [30]. This is essentially an application of the Lagrangian description in fluid mechanics [40, 41], as opposed to the Eulerian, to the Langevin equation for the density field. The label-variable method allows us, on one hand, to calculate four-point correlations explicitly. On the other hand, we demonstrate that an MCT-like nonlinear theory for the fluctuation of $1 / \rho$ can be developed in a field-theoretical style, without violating the fluctuation-dissipation theorem (FDT). This is possible because the problem of the multiplicative noise is naturally resolved by virtue of the label variable. Using this version of $\mathrm{MCT}$, we find the contribution of the memory term to give a finite-time correction to the asymptotic Hahn-Kärger-Kollman law (1.4), visible as a finite intercept of the asymprotic straight line on the $\sqrt{t}$-axis in the inset in Fig. 1.

We obtain four-point correlations by generalizing the calculation of MSD [30] to the two-particle displacement correlation $(2 \mathrm{pDC})$,

$$
\left\langle R_{i} R_{j}\right\rangle=\left\langle\left[X_{i}(t)-X_{i}(0)\right]\left[X_{j}(t)-X_{j}(0)\right]\right\rangle .
$$

Quantities analogous to $2 \mathrm{pDC}$ have been studied by a number of researchers with numerical data from molecular dynamics of glassy liquids [42 45] and with linear theories of generalized elastic model for systems such as fluctuating membranes [46, 47], and 2pDC is also the main ingredient of the theory of $\chi_{4}$ for elastic waves by Toninelli et al. 18]. Nevertheless, analytical calculations of $2 \mathrm{pDC}$ for "bondless" particle systems have never been reported. Here we calculate $2 \mathrm{pDC}$ analytically in terms of generalizable and liquid-oriented concepts, so that the theory could be extended to truly bondless systems in the near future. At first, we calculate $2 \mathrm{pDC}$ as a function of the elapsed time $t$ and some properly defined label distance which coincides with $|i-j|$ in the one-dimensional cases; and subsequently, we show it to be re-expressible as a function of $t$ and the initial distance $\tilde{d}=X_{j}(0)-X_{i}(0)$, which we denote with $X_{R}(\tilde{d}, t)$. For $i=j$, Eq. (1.6) reduces to MSD. The two-particle correlation, $\left\langle R_{i} R_{j}\right\rangle$ with $i \neq j$, provides an intuitive form of four-point correlation in comparison to $\chi_{4}$; the $2 \mathrm{pDC}$ with $i \neq j$ vanishes for free Brownian particles and, for SFD, evidences the cluster size that behaves like $\rho_{0} \sqrt{D_{\mathrm{c}} t}$, accounting for the slow diffusion. In addition, from the knowledge of $\left\langle R_{i} R_{j}\right\rangle$, we can perform a fully analytical calculation for the self part of $\chi_{4}$, denoted with $\chi_{4}^{\mathrm{S}}$. Reflecting the eternity of the onedimensional cages, the long-time limiting value, $\chi_{4}^{\mathrm{S}}(+\infty)$, is finite, as will be shown in Eq. (5.17).

The paper is organized as follows: After summarizing in Sec. II the idea of the continuous label-variable method and some of its results, we apply it to the cal- 
culation of the two-particle displacement correlation in Sec. III. Strictly speaking, what we present in Sec. III] is not $\left\langle R_{i} R_{j}\right\rangle$ itself but its continuum equivalent, calculated theoretically for the long-time regime. Subsequently, in Sec.IV]we demonstrate a systematic and FDT-preserving derivation of an MCT-like equation in the Lagrangian description. The "Lagrangian" MCT equation provides us with the finite-time correction to MSD and the twoparticle displacement correlation. With this finite-time correction taken into account, two different forms of fourpoint correlation functions are calculated in Sec. $\mathrm{V}$, one is $X_{R}(\tilde{d}, t)$ and the other is $\chi_{4}^{\mathrm{S}}(t)$. We will discuss in Sec.VI how the collective dynamics is represented by $\chi_{4}^{\mathrm{S}}(t)$, what is the relation between the theories of SFD in the past and the present one, and whither the method of the Lagrangian MCT may guide us in the future. Section VII is allotted for concluding remarks.

\section{CONTINUUM THEORY OF SINGLE-FILE DIFFUSION}

Let us begin with summarizing our previous results on MSD for SFD [30]. By "continuum theory" we mean that the theory is formulated in terms of some hydrodynamic quantity such as the density $\rho$, rather than direct treatment of the particles. Our idea consists in adoption of the continuous label variable $\xi$, which we take instead of the position $x$ as the independent variable, and we also change the dependent variable from the density $\rho(x, t)$ to the fluctuation of the particle interval, denoting it with $\psi(\xi, t)$. On the basis of the correlation of $\psi$ calculated for the long-time regime, we can re-derive Eq. (1.4).

Since our label-variable method is intended as a reformulation of MCT, we start from essentially the same Langevin equation as in the field-theoretical formulation of MCT for dense colloidal suspension [48]. The Langevin equation, derived from Eq. (1.2) for the density $\rho(x, t)=\sum_{j} \rho_{j}$ with $\rho_{j}=\delta\left(x-X_{j}(t)\right)$ and its flux $Q=\sum_{j} \rho_{j} \dot{X}_{j}$, is given as follows:

$$
\begin{aligned}
& \partial_{t} \rho+\partial_{x} Q=0, \\
& Q=-D\left(\partial_{x} \rho+\frac{\rho}{k_{\mathrm{B}} T} \partial_{x} U\right)+\sum_{j} \rho_{j}(x, t) f_{j}(t), \\
& U=U[\rho](x)=\int \mathrm{d} x^{\prime} V\left(x-x^{\prime}\right) \rho\left(x^{\prime}\right) .
\end{aligned}
$$

By eliminating $Q$ and introducing

$$
f_{\rho}(x, t)=-\partial_{x} \sum_{j} \rho_{j}(x, t) f_{j}(t),
$$

we write down the equation for $\rho(x, t)$ as

$$
\partial_{t} \rho(x, t)=D \partial_{x}\left(\partial_{x} \rho+\frac{\rho}{k_{\mathrm{B}} T} \partial_{x} U\right)+f_{\rho}(x, t)
$$

with $\partial_{x} U=\partial_{x} U[\rho](x)$ and

$$
\left\langle f_{\rho}(x, t) f_{\rho}\left(x^{\prime}, t^{\prime}\right)\right\rangle=2 D \partial_{x} \partial_{x^{\prime}} \rho(x, t) \delta\left(x-x^{\prime}\right) \delta\left(t-t^{\prime}\right) .
$$

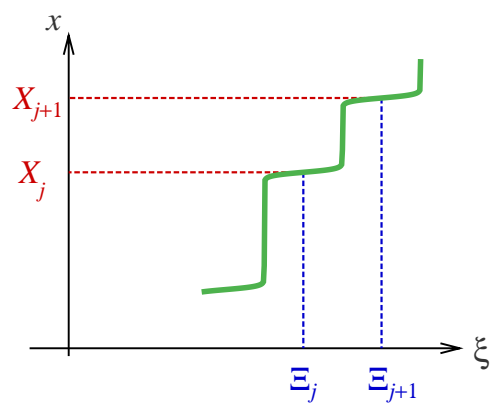

FIG. 2: (Color online) A schematic view of the mapping from $\xi$ to $x$, given by inverting the function $\xi=\xi(x, t)$ in Eq. 2.6), with $t$ fixed arbitrarily. The label and the position of the $j$-th particle is denoted by $\Xi_{j}$ and $X_{j}$, respectively.

Note the presence of $\rho(x, t)$ on the right-hand side of Eq. (2.3): the noise is multiplicative [48 52]. A linear version of Eq. (2.2) is sometimes referred to as the diffusionnoise equation [53], which was used to describe collective dynamics of a single-file system [54, 55].

As the particles have the excluded volume effect and therefore cannot overlap, the barrier expressed by $V$ must be infinitely high. In the one-dimensional system, this barrier acts as a topological constraint, or the "no overtaking" rule in plain words, which keeps the order of the particles. In MCT formulated for coarse-grained $\rho$, however, $V$ must be replaced with a finite effective potential, in a manner analogous to the dynamical densityfunctional theory [56, 57]. As a result, MCT fails to incorporate the "no overtaking" effect of $V$ properly. An asymptotic analysis of MCT for the long-time limit shows that a certain mathematical feature of the MCT memory kernel (the presence or the absence of the zero-frequency singularity in Laplace transform of $M_{\mathrm{S}}$ ) determines the asymptotic behavior of $\left\langle R^{2}\right\rangle$. The memory kernels, $M$ and $M_{\mathrm{S}}$, ultimately decay exponentially on the liquid side of the theory, though anomalous diffusion may occur transiently near the MCT transition point [58, 59]. As a corollary, the one-dimensional version of Eq. (1.5) gives the normal diffusion, contradicting to Eq. (1.4) and suggesting that the mathematical feature of the MCT kernel is not compatible with the "no overtaking" rule [38, 39].

Thus the difficulty is located in the memory kernel $M_{\mathrm{S}}$ in Eq. (1.5). Consequently, for an MCT-based nonlinear theory of SFD, there is a choice between two strategies. The first one consists in amending the memory kernel $M_{\mathrm{S}}$, so that it has a proper singularity; this is the strategy developed by Fedders [22] and Abel et al. [34]. Alternatively, one may totally dispense with $M_{\mathrm{S}}$, using only the collective MCT equation corresponding to Eq. (1.1), and replacing the tagged-particle MCT equation (1.5) by something that gives $\left\langle R^{2}\right\rangle$ without time integral. Here we choose this alternative, which becomes possible by importing the concept of Lagrangian correlation from the theory of fluid turbulence [60 62]. The idea consists in adoption of the continuous label variable, which we denote with $\xi$.

Though it is popular in continuum mechanics to take 
the initial position of each material element to label it, here we define $\xi$ in a different way, avoiding to trace the whole history of the system back to the initial configuration. We construct the label function $\xi=\xi(x, t)$ so as to satisfy the following three requirements:

1. The label should satisfy the convective equation

$$
\left(\rho \partial_{t}+Q \partial_{x}\right) \xi(x, t)=0 .
$$

2. The label should be related to the snapshot of $\rho$ and $Q$ in such a way that the continuity equation (2.1a) is satisfied.

3. The function needs to be invertible, in the sense that a mapping from $(\xi, t)$ to $x=x(\xi, t)$ should exist.

To satisfy the second requirement, we utilize the fact that Poincaré's lemma [63] is applicable to the continuity equation (2.1a), which guarantees the existence of $\xi$ such that

$$
\partial_{x} \xi(x, t)=\rho, \quad \partial_{t} \xi(x, t)=-Q
$$

in fact, a solution to Eq. (2.5) is explicitly given by

$$
\xi=\xi(x, t)=\int_{X_{0}(t)}^{x} \rho\left(x^{\prime}, t\right) \mathrm{d} x^{\prime}+\text { const. }
$$

Then it is straightforward to verify that all the three requirements are satisfied. To be precise, since $\rho$ consists of a sum of delta functions, the integral in Eq. (2.6) gives a multiple step function (see Fig. 2), which needs to be slightly smoothed to justify the single-valuedness and the invertibility. Although this smoothing could be interpreted physically as a consequence of coarse-graining, here we refrain from involving ourselves with such a delicate matter and regard the smoothing simply as a mathematical regularization. We define $\Xi_{j}=\xi\left(X_{j}(t), t\right)$ for $j \in \mathbb{Z}$, taking it for granted that the particles are numbered consecutively; then, with the smoothing and the integral constant tuned appropriately, we have $\Xi_{j}=j$.

Using the label variable $\xi$ as the spatial coordinate, now we rewrite the Langevin equation (2.1). The chain rule for the differential operators gives

$$
\partial_{x}=\frac{\partial \xi}{\partial x} \partial_{\xi}=\rho \partial_{\xi}, \quad\left(\partial_{t} \cdot\right)_{x}=\left(\partial_{t} \cdot\right)_{\xi}-Q \partial_{\xi}
$$

As a kinematic relation that replaces Eq. (2.1a), from the identity $\partial_{t} \partial_{\xi} x=\partial_{\xi} \partial_{t} x$ we find

$$
\partial_{t}\left[\frac{1}{\rho(\xi, t)}\right]=\partial_{\xi}\left(\frac{Q}{\rho}\right) \text {. }
$$

Then, rewriting Eq. (2.1b) with $\partial_{\xi}$ and substituting it into Eq. (2.7), we obtain

$$
\begin{aligned}
\partial_{t}\left[\frac{1}{\rho(\xi, t)}\right]= & -D \partial_{\xi}\left(\partial_{\xi} \rho+\frac{\rho}{k_{\mathrm{B}} T} \partial_{\xi} U\right) \\
& +\partial_{\xi} \sum_{j} \delta\left(\xi-\Xi_{j}\right) f_{j}(t) \\
= & -D \partial_{\xi}\left[\partial_{\xi} \rho+2 \sinh \left(\rho \sigma \partial_{\xi}\right) \rho\right]+f_{\mathrm{L}}(\xi, t),
\end{aligned}
$$

where $V$ is replaced with the effective potential as before, and $f_{\mathrm{L}}$ satisfies

$$
\left\langle f_{\mathrm{L}}(\xi, t) f_{\mathrm{L}}\left(\xi^{\prime}, t^{\prime}\right)\right\rangle=2 D \partial_{\xi} \partial_{\xi^{\prime}} \sum_{i} \delta\left(\xi-\Xi_{i}\right) \delta\left(\xi-\xi^{\prime}\right) \delta\left(t-t^{\prime}\right) .
$$

Having changed the independent variables from $(x, t)$ to $(\xi, t)$, we change the dependent variable as well. Introducing the fluctuation of the particle interval,

$$
\psi=\psi(\xi, t)=\frac{\rho_{0}}{\rho(\xi, t)}-1
$$

and its Fourier modes

$$
\check{\psi}(k, t)=\frac{1}{N} \int \mathrm{d} \xi e^{\mathrm{i} k \xi} \psi(\xi, t) \quad\left(\frac{k}{2 \pi / N} \in \mathbb{Z}\right)
$$

we rewrite Eq. (2.8) in the form

$$
\begin{array}{r}
\partial_{t} \check{\psi}(k, t)=-D_{*} k^{2}\left(1+\frac{2 \sin \rho_{0} \sigma k}{k}\right) \check{\psi}(k, t) \\
+\sum_{p+q+k=0} \mathcal{V}_{k}^{p q} \check{\psi}(-p, t) \check{\psi}(-q, t)+O\left(\check{\psi}^{3}\right) \\
+\rho_{0} \check{f}_{\mathrm{L}}(k, t)
\end{array}
$$

with $D_{*}=\rho_{0}^{2} D$ and

$$
\begin{aligned}
& \mathcal{V}_{k}^{p q} \\
= & D_{*} k^{2}\left(1+\frac{k}{p q} \sin \rho_{0} \sigma k+\frac{p}{k q} \sin \rho_{0} \sigma p+\frac{q}{k p} \sin \rho_{0} \sigma q\right) .
\end{aligned}
$$

The summation is taken over all $(p, q)$ satisfying the triad condition $p+q+k=0$ for given $k$ [64]. As for the statistics of the random force term, Eq. (2.9) is re-expressed as

$$
\rho_{0}^{2}\left\langle\check{f}_{\mathrm{L}}(k, t) \check{f}_{\mathrm{L}}\left(-k^{\prime}, t^{\prime}\right)\right\rangle=\frac{2 D_{*}}{N} k^{2} \delta_{k k^{\prime}} \delta\left(t-t^{\prime}\right)
$$

see Endnote 65]. We also note that the linearization of Eq. (2.12), corresponding to harmonization of the effective interaction between the particles [18, 36, 45, 66], coincides with the one-dimensional version of EdwardsWilkinson equation [33, 67] and also with the Rouse model [68].

To calculate MSD without employing $M_{\mathrm{S}}$, we developed a formula for it in terms of the correlation $\left\langle\psi(\xi, t) \psi\left(\xi^{\prime}, 0\right)\right\rangle$, or its Fourier transform $\left\langle\check{\psi}(k, t) \check{\psi}\left(-k^{\prime}, 0\right)\right\rangle$. In Fourier representation, the formula reads as follows:

$$
\left\langle R^{2}\right\rangle=\frac{L^{4}}{\pi N^{2}} \int_{-\infty}^{\infty} \frac{\check{C}(k, 0)-\check{C}(k, t)}{k^{2}} \mathrm{~d} k
$$

where 69]

$$
\check{C}(k, t)=\frac{N}{L^{2}}\langle\check{\psi}(k, t) \check{\psi}(-k, 0)\rangle .
$$

In Sec. III this formula will be re-derived as a special case of Eq. (3.2). 
Once the formula (2.15) is derived, all depends on the knowledge of $\breve{C}$. In particular, the long-time behavior of MSD is found by the linear analysis of Eq. (2.12). From the linearized equation, the correlation $\check{C}$ is calculated as

$$
\check{C}(k, t)=\frac{S}{L^{2}} e^{-\left(D_{*} / S\right) k^{2} t}, \quad S=S(k) \simeq S(0)
$$

with the aid of Eq. (2.14). On the other hand, linear analysis implies that the difference between the Eulerian and Lagrangian descriptions vanishes up to a trivial rescaling of the variables. Thus the linearized version of Eq. (2.15) corresponds to the approximate formula by Alexander and Pincus [23],

$$
\left\langle R^{2}\right\rangle \simeq \text { const. } \times \int_{-\infty}^{\infty} \frac{F(q, 0)-F(q, t)}{q^{2}} \mathrm{~d} q,
$$

which evidently reproduces the subdiffusion law in Eq. (1.4). For the special case of pointlike particles $(\sigma=0), S$ in Eq. 2.17) is replaced with unity; more generally, the linear formulation is also readily applicable to systems with arbitrary interaction potential and, in this sense, turns out to be equivalent to the theory of Kollmann [26]. A refined treatment, including the effect of the nonlinear term on $\check{C}$, requires Eq. (2.15) instead of Eq. (2.18). We refer to Eq. (2.15) as the modified Alexander-Pincus formula: the modification consists in the adoption of the Lagrangian description.

In the linear (Edwards-Wilkinson) case, a $n_{\mathrm{d}^{-}}$ dimensional version of Eq. (2.18) has appeared in the literature [18, 70, 71]; we will discuss it later in Sec. VI. calling attention to some delicate points about the extension to the $n_{\mathrm{d}}$-dimensional liquid dynamics.

\section{FOUR-POINT CORRELATION}

\section{A. Cooperative motion in SFD}

The slow dynamics in SFD is associated with collective motion of the particles. This collective motion, obtained by numerical integration of Eq. (1.2), is depicted in Fig. 3. In the numerical calculations (see Appendix A for details), after the system has reached the thermal equilibrium, we choose some instance as $t=0$ and record the "initial" position of each particle, say, $X_{i}(0)$. To produce Fig. 3(a), at $t=2^{n} \times 10 \sigma^{2} / D$ (with $n=1,2, \ldots$ ) we measured the displacement $R_{i}(t)=X_{i}(t)-X_{i}(0)$ for each $i$. If $R_{i}(t)>5 \sigma$, we mark the position of the particle with a circle $(\bigcirc)$; if $R_{i}(t)<-5 \sigma$, we mark it with a cross $(\times)$. As the time difference $t$ increases, a string of the same kind of symbol is formed, expressing a cluster of particles in cooperative motion. While Fig. 3(a) presents a close-up for a relatively limited time span, a long shot up to $t=10^{6} \sigma^{2} / D$ is shown as Fig. 3(b). Formation of large clusters in cooperative motion is visible. By the time difference $t=10^{6} \sigma^{2} / D$, a typical cluster reaches the size of several hundred particles, occupying a length on the order of $10^{3} \sigma$ (as each particle is assigned a space of $\left.1 / \rho_{0}=4 \sigma\right)$.
Quantitative description of this collective motion requires some four-point correlation functions, such as those used for the analysis of dynamical heterogeneity in glassy systems. In regard to SFD, it seems especially natural to consider the four-point correlation to deal with the topological constraint of the "no passing" rule, as the requirement that two world lines should not intersect involves four points in the space-time, namely $X_{i}(0), X_{j}(0), X_{i}(t)$, and $X_{j}(t)$. This is why Miyazaki and Yethiraj [37] needed to calculate a four-point correlation $[G(1,2 ; 3,4)$ in their notation] to study the entanglements of rod polymers within the framework of liquid state theory, and why Abel et al. 34], to improve on the conventional MCT which fails in reproducing the subdiffusive law for SFD, had to examine the irreducible four-point propagator (denoted with $\chi^{\text {irr }}$ ).

Here we demonstrate that the label-variable method is also capable of calculating a kind of four-point correlation, in the form of two-particle displacement correlation. Though it was originally presented as $\left\langle R_{i} R_{j}\right\rangle$ in Eq. (1.6), there is no trouble in replacing the particle numbering with the label variable. Then, our task is derivation of a formula to calculate $\left\langle R(\xi, t) R\left(\xi^{\prime}, t\right)\right\rangle$ with $R(\xi, t)=x(\xi, t)-x(\xi, 0)$, which will be shown in the next subsection.

\section{B. Two-Particle Displacement Correlation}

Aiming for an analytical expression for $\left\langle R(\xi, t) R\left(\xi^{\prime}, t\right)\right\rangle$ in SFD, let us extend Eq. (2.15) so that we can calculate $\left\langle R(\xi, t) R\left(\xi^{\prime}, t\right)\right\rangle$ from the correlation $\check{C}$ defined by Eq. (2.16). We start with noticing that $Q / \rho$ stands for the velocity, whose integral in regard to $t$ gives the displacement of the particle labelled with $\xi$ :

$$
R(\xi, t)=\int_{0}^{t} \mathrm{~d} t^{\prime} \frac{Q\left(\xi, t^{\prime}\right)}{\rho\left(\xi, t^{\prime}\right)}
$$

Into this equation we substitute $Q / \rho=\partial_{\xi}^{-1} \partial_{t}(1 / \rho)$, obtained from Eq. (2.7) upon integration over $\xi$, to find

$$
\begin{aligned}
R(\xi, t) & =\left.\partial_{\xi}^{-1}\left(\frac{1+\psi}{\rho_{0}}\right)\right|_{0} ^{t} \\
& =\frac{1}{\rho_{0}} \sum_{k} \frac{e^{-\mathrm{i} k \xi}}{-\mathrm{i} k}[\check{\psi}(k, t)-\check{\psi}(k, 0)] .
\end{aligned}
$$

Subsequently, we multiply Eq. (3.1) by its duplicate with $(\xi, k)$ changed to $\left(\xi^{\prime},-k^{\prime}\right)$, and take the statistical average. The double summation on the right-hand side reduces to the single one, if we assume that the contribution from the terms with $k \neq k^{\prime}$ vanishes. This is true in the linear case, and also seems to be justifiable for nonlinear cases within the framework of the direct-interaction approximation (explained later). Thus we obtain a for- 
(a)

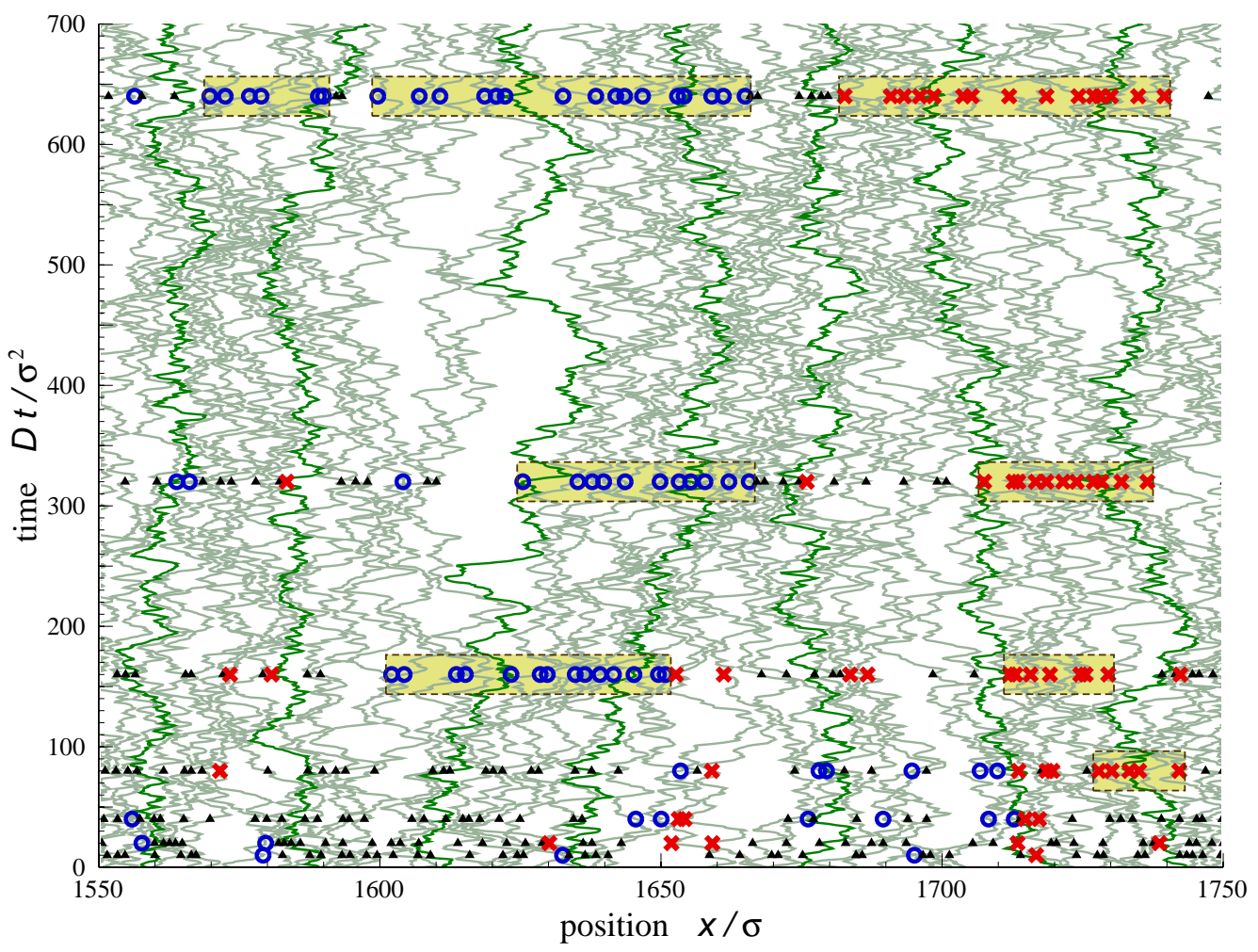

(b)

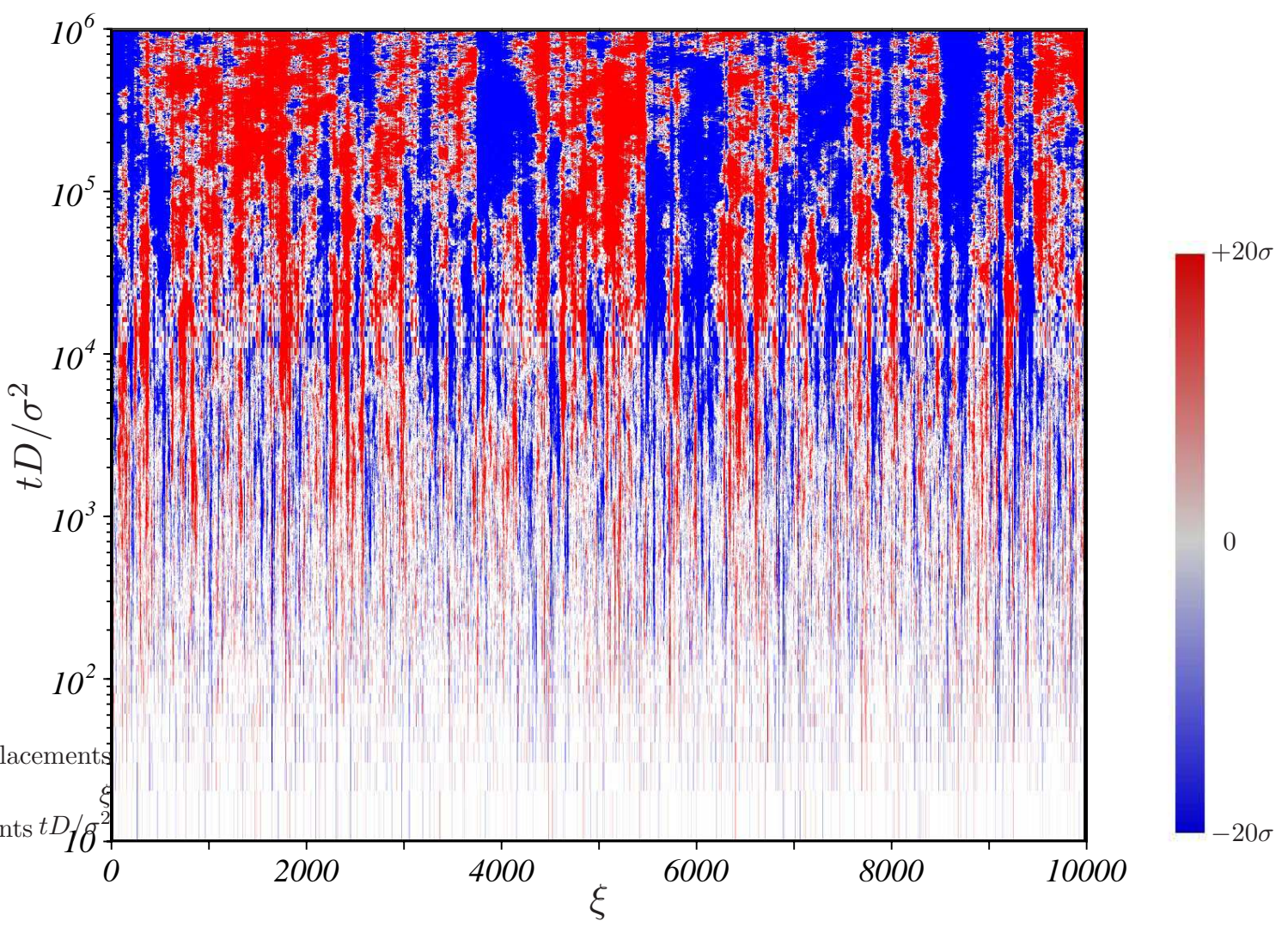

FIG. 3: (Color online) Clusters in cooperative motion visualized in space-time diagrams of particles in SFD, calculated for $\rho_{0}=N / L=0.25 \sigma^{-1}$. (a) Worldlines of particles in the $(x, t)$-plane. The symbols $\bigcirc$ and $\times$ mark particles displaced (by more than $5 \sigma$ ) rightward and leftward, respectively. The unmoving particles are indicated with small triangles. Each cluster in cooperative motion, involving 5 particles at least, is highlighted in a box. (b) The displacement $R$ depicted in gray scale (blue-to-red scale online) as a function of $\xi$ and $t$. Unmoving particles are shown in white. 
mula allowing us to calculate $2 \mathrm{pDC}$ from $\langle\psi \psi\rangle$ :

$$
\begin{aligned}
& \left\langle R(\xi, t) R\left(\xi^{\prime}, t\right)\right\rangle \\
& \quad=\frac{L^{4}}{\pi N^{2}} \int_{-\infty}^{\infty} \mathrm{d} k e^{-\mathrm{i} k\left(\xi-\xi^{\prime}\right)} \frac{\check{C}(k, 0)-\check{C}(k, t)}{k^{2}},
\end{aligned}
$$

with $\check{C}(k, t)$ defined by Eq. (2.16). Note that Eq. (3.2) includes the modified Alexander-Pincus formula (2.15) as a special case with $\xi=\xi^{\prime}$, as it ought to be. In this sense, the formula (3.2) could be referred to as the extended Alexander-Pincus formula. We emphasize that Eq. (3.2) does not rely on smallness of deformation, nor it requires such kind of approximation at all, as long as the Lagrangian description is strictly followed.

\section{Calculation of 2pDC: linear approximation}

For $\check{C}$ in Eq. (2.17) calculated from the linear approximation of Eq. (2.12), the extended Alexander-Pincus formula (3.2) gives

$$
\begin{aligned}
& \left\langle R(\xi, t) R\left(\xi^{\prime}, t\right)\right\rangle \\
= & \frac{2 S}{\rho_{0}} \sqrt{\frac{D_{\mathrm{c}} t}{\pi}} \exp \left[-\frac{\left(\xi-\xi^{\prime}\right)^{2}}{4 \rho_{0}^{2} D_{\mathrm{c}} t}\right]-\frac{S}{\rho_{0}^{2}}\left|\xi-\xi^{\prime}\right| \operatorname{erfc} \frac{\left|\xi-\xi^{\prime}\right|}{2 \rho_{0} \sqrt{D_{\mathrm{c}} t}}
\end{aligned}
$$

reproducing Eq. (B3) in Ref. [30]; the same result was reported in regard to the generalized elastic model [47]. This is expressible in terms of a similarity variable

$$
\theta=\frac{\xi-\xi^{\prime}}{2 \rho_{0} \sqrt{D_{\mathrm{c}} t}}
$$

as

$$
\frac{\left\langle R(\xi, t) R\left(\xi^{\prime}, t\right)\right\rangle}{\sigma \sqrt{D_{\mathrm{c}} t}}=\frac{2 S}{\rho_{0} \sigma}\left(\frac{e^{-\theta^{2}}}{\sqrt{\pi}}-|\theta| \operatorname{erfc}|\theta|\right)=\varphi(\theta) .
$$

From this similarity solution we can read the dynamical correlation length

$$
\lambda=\lambda(t)=2 \sqrt{D_{\mathrm{c}} t}
$$

indicating the size $\lambda$ of a cluster in a cooperative motion. We have already seen such clusters in Fig. 3, though care should be taken in regard to the difference that Eq. (3.5) is a statistical law while Figs. 3(a) and (b) present a single run, and only a small portion of it is shown in Fig. 3(a). The dynamical correlation length $\lambda$ in Eq. (3.5) is the diffusive one (with the exponent $1 / 2$ ), which may occur also in different contexts, such as roughening of growing surfaces 72 and kinetically constrained models of defectmediated glassy dynamics [18].

Equation (3.3) is compared with statistical results of particle simulations in Fig. 4. Except for the transient behavior slightly visible for $t=100 \sigma^{2} / D$, all of the simulation results are consistent with Eq. (3.3). The transient behavior can be studied by taking the nonlinear terms in

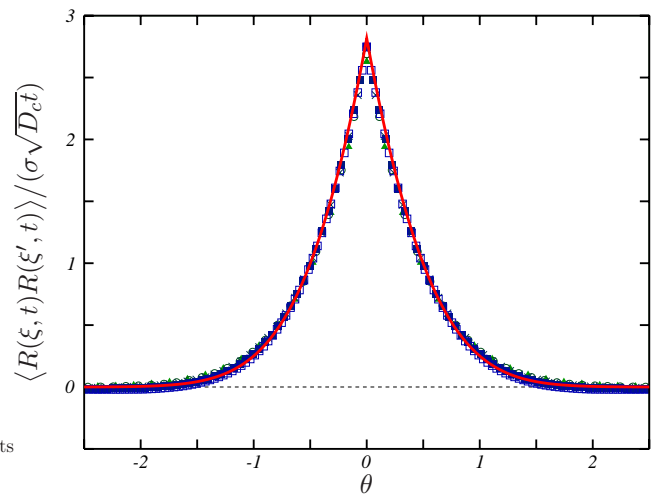

FIG. 4: (Color online) Comparison of Eq. (3.3) with the computed $2 \mathrm{pDC}$ in the same system as in Fig. 1 $(N=$ $2^{15}=32768, \rho_{0}=N / L=0.25 \sigma^{-1}$ ). The solid (red) line shows the self-similar solution in Eq. 3.3 , while the simulation data are plotted with symbols: solid triangles $(\boldsymbol{\Lambda})$ for $t=100 \sigma^{2} / D$, open circles $(\bigcirc)$ for $t=200 \sigma^{2} / D$, crosses $(\times)$ for $t=400 \sigma^{2} / D$, open squares $(\square)$ for $t=800 \sigma^{2} / D$, and solid squares (ם) for $t=1600 \sigma^{2} / D$.

Eq. (2.12) into account, which will be discussed in the next section.

The variable for the horizontal axis of Fig. 4 requires some consideration. As a quantity corresponding to $\xi-\xi^{\prime}$, here we have taken the distance in the label numbering, say, $|i-j|$ between the $i$-th and $j$-th particle. Though adequate in the present case, this is not convenient for extensions to multidimensional cases [44, 45], because the result may depend on the way of numbering. A reasonable alternative in the particle simulation is the initial distance such as $X_{i}(0)-X_{j}(0)$, which can be compared with the theoretical prediction by assuming that $\left(\xi-\xi^{\prime}\right) / \rho_{0}$ corresponds to $X_{i}(0)-X_{j}(0)$ after statistical averaging. Numerical calculations show that this is indeed valid as far as the long-time behavior is concerned, but transiently there are additional modifications due to triple correlations such as $\langle\breve{\psi}(-p, 0) \breve{\psi}(-q, 0) \breve{\psi}(-k, t)\rangle$. Before discussing these modifications, let us develop a nonlinear closure theory for $\check{C}$, which introduces the entropic nonlinearity into the theory of SFD, making the triple correlation available as a byproduct.

\section{NONLINEAR THEORY FOR FINITE-TIME EFFECTS}

\section{A. Inclusion of nonlinearity: DIA for SFD}

The expression for $2 \mathrm{pDC}$ in Eq. (3.3) is based on the linear approximation to Eq. (2.12), which is only asymptotically valid for sufficiently long time. For finite values of $t$, there should be a correction to Eq. (3.3) describing the transient behaviors of $2 \mathrm{pDC}$ and MSD; to find this correction, now we develop a nonlinear closure theory for the correlation $\check{C}$.

In short, what we present here and in the next subsection is a systematic derivation of $\mathrm{MCT}$ equation for 
$\check{C}$. Except for some minor (but important) differences, this is analogous to the attempt of a number of authors [49, 73, 74] who tried to re-derive MCT equation with the Martin-Siggia-Rose (MSR) formalism [75]. They were obstructed by the problem of inconsistency with FDT: this difficulty is inevitable for dense colloidal suspensions or supercooled liquids, as long as one uses the standard MSR formalism with the plain $\hat{\rho}(\mathbf{k}, t)$ as the field variable [50 52, 76]. Only some special classes of Langevin equations are free from this difficulty. Two such classes are known: one is the class of models whose nonlinearity comes from the gradient of the thermodynamic potential (entropy) alone, such as the $p$-spin model, referred to as "Class I" by Miyazaki and Reichman [49]; and the other one (called "Class II") is exemplified by liquid models with Gaussian approximation to the entropy [49, 74]. Fortunately, our equation for $\breve{\psi}$ belongs to Class I and therefore consistency with FDT is expected.

Let us return to the nonlinear Langevin equation (2.12) governing $\breve{\psi}$, with the random force statistics in Eq. (2.14). The correlation $\breve{C}$ is then subject to an equation containing triple correlations:

$$
\begin{aligned}
\left(\partial_{t}\right. & \left.+\frac{D_{*}}{S} k^{2}\right) \check{C}(k, t) \\
& =\frac{N}{L^{2}} \sum_{p+q+k=0} \mathcal{V}_{k}^{p q}\langle\check{\psi}(-p, t) \check{\psi}(-q, t) \check{\psi}(-k, 0)\rangle,
\end{aligned}
$$

with the $O\left(\check{\psi}^{3}\right)$ term in Eq. (2.12) discarded, and with $\sum_{p+q+k=0}$ denoting the same summation over $(p, q)$ as in Eq. (2.12). Note the absence of $\left\langle\check{f}_{\mathrm{L}} \check{\psi}\right\rangle$ on the righthand side; this term vanishes because $f_{\mathrm{L}}$ is not multiplicative, which means that $\left\langle\check{f}_{\mathrm{L}}(k, t) \check{f}_{\mathrm{L}}\left(-k^{\prime}, t^{\prime}\right)\right\rangle$ is independent of the $\check{\psi}$ 's according to Eq. (2.14). To provide a closure to this equation, we apply the formalism of direct-interaction approximation (DIA) 60 62, 77, 78]. The procedure of the calculation is essentially the same as that in Ref. 79] and is explained briefly in Appendix[B. As a result, we obtain a set of equations:

$$
\begin{aligned}
\left(\partial_{t}+\frac{D_{*}}{S} k^{2}\right) \check{C}(k, t)= & \int_{t_{0}}^{t} \mathrm{~d} t^{\prime} M_{G}\left(k, t-t^{\prime}\right) \check{C}\left(k,\left|t^{\prime}\right|\right) \\
& +\int_{t_{0}}^{0} \mathrm{~d} t^{\prime} M_{C}\left(k, t-t^{\prime}\right) \bar{G}\left(-k,-t^{\prime}\right)
\end{aligned}
$$$$
\left(\partial_{t}+\frac{D_{*}}{S} k^{2}\right) \bar{G}(k, t)=\int_{0}^{t} \mathrm{~d} t^{\prime} M_{G}\left(k, t-t^{\prime}\right) \bar{G}\left(k, t^{\prime}\right)
$$

for the correlation $\check{C}$ and

$$
\bar{G}\left(k, t-t^{\prime}\right)=\left\langle G\left(k, t ; k, t^{\prime}\right)\right\rangle=\left\langle\frac{\delta \check{\psi}(k, t)}{\delta \check{\psi}\left(k, t^{\prime}\right)}\right\rangle,
$$

with $t_{0}(<0)$ denoting the time at which the "direct interactions" are switched off, and $M_{G}$ and $M_{C}$ are memory kernels given by

$$
\begin{aligned}
& M_{G}(k, s)=\frac{4 L^{2}}{N} \sum_{p+q+k=0} \mathcal{V}_{k}^{p q} \mathcal{V}_{q}^{p k} \check{C}(p, s) \bar{G}(-q, s), \\
& M_{C}(k, s)=\frac{2 L^{2}}{N} \sum_{p+q+k=0}\left(\mathcal{V}_{k}^{p q}\right)^{2} \check{C}(p, s) \check{C}(q, s) .
\end{aligned}
$$

Note that, in the present case, the propagator (Kraichnan's response function) $\bar{G}$ is essentially equivalent to the response function to an externally applied probe force, because the random forcing term $\rho_{0} \tilde{f}_{\mathrm{L}}$ in Eq. (2.12) is not multiplicative but additive.

In regard to the choice of $t_{0}$, we consider two possibilities. Choosing $t_{0} \rightarrow-0$ would admit a solution of the form $\check{C}(t)=\bar{G}(t) \check{C}(0)$, which corresponds to the "Class II" approximation; we do not take this choice, as this would require the Langevin equation to belong to Class I and II simultaneously, leading to a result that is either trivial or inconsistent with FDT. Instead, we take $t_{0} \rightarrow-\infty$, so that Eqs. (4.1) and (4.2) become identical to the standard one-loop result of the MSR formalism 75.

\section{B. Label-based MCT equation for SFD}

In principle, Eqs. (4.1) and (4.2) with the initial conditions

$$
\check{C}(k, 0)=\frac{S(k)}{L^{2}}, \quad \bar{G}(k, 0)=1
$$

should suffice for determination of $\check{C}$ and $\bar{G}$. However, as soon as we start to calculate them in this straightforward way, we find us confronted with difficulties. Since we have $t_{0} \rightarrow-\infty$, the equations are acausal. Besides, the memory terms seem to suffer ultraviolet divergence. To elude these difficulties, we demonstrate that one of the two equations can be replaced with a simpler relation between $\check{C}$ and $\bar{G}$, later shown as Eq. (4.7). We start with differentiating Eq. (4.1) in regard to $t$ and adding it to Eq. (4.2) multiplied by $\alpha_{0} k^{2}$ with some constant $\alpha_{0}$, to write an equation for $\partial_{t} \check{C}+\alpha_{0} k^{2} G$ :

$$
\begin{aligned}
\left(\partial_{t}+\frac{D_{*}}{S} k^{2}\right)\left[\partial_{t} \check{C}(k, t)+\alpha_{0} k^{2} G(k, t)\right]= & \int_{0}^{t} \mathrm{~d} t^{\prime} M_{G}\left(k, t-t^{\prime}\right)\left[\partial_{t} \check{C}\left(k, t^{\prime}\right)+\alpha_{0} k^{2} G\left(k, t^{\prime}\right)\right] \\
& +\int_{t_{0}}^{0} \mathrm{~d} t^{\prime}\left\{M_{G}\left(k, t-t^{\prime}\right) \partial_{t^{\prime}} \check{C}\left(k,-t^{\prime}\right)-\left[\partial_{t^{\prime}} M_{C}\left(k, t-t^{\prime}\right)\right] \bar{G}\left(-k,-t^{\prime}\right)\right\},
\end{aligned}
$$


with $t_{0} \rightarrow-\infty$ taken into account. The second term on the right-hand side includes $\mathcal{V}$ 's through the memory kernels, which we rewrite by substituting Eqs. (4.3) and (4.4). Subsequently, introducing $W$ by $\mathcal{V}_{k}^{p q}=D_{*} k^{2} W_{k p q}$ and making use of the symmetry of $W$ [see Eq. [2.13)], after some algebraic manipulation, we find

[the integrand in the 2nd term on RHS of Eq. 4.5)]

$$
\begin{aligned}
= & \frac{4 L^{2}}{N} D_{*}^{2} k^{2} \sum W_{k p q}^{2}\left[q^{2} \bar{G}\left(-q, t-t^{\prime}\right) \partial_{t^{\prime}} \check{C}\left(k,-t^{\prime}\right)-k^{2} \bar{G}\left(k,-t^{\prime}\right) \partial_{t^{\prime}} \check{C}\left(q, t-t^{\prime}\right)\right] \\
= & \frac{4 L^{2}}{N} D_{*}^{2} k^{2} \sum W_{k p q}^{2} \times \\
& \quad\left\{q^{2} \bar{G}\left(-q, t-t^{\prime}\right)\left[\partial_{t^{\prime}} \check{C}\left(k,-t^{\prime}\right)-\alpha_{0} k^{2} \bar{G}\left(k,-t^{\prime}\right)\right]-k^{2} \bar{G}\left(k,-t^{\prime}\right)\left[\partial_{t^{\prime}} \check{C}\left(-q, t-t^{\prime}\right)-\alpha_{0} k^{2} \bar{G}\left(-q, t-t^{\prime}\right)\right]\right\} .
\end{aligned}
$$

Then Eq. (4.5) can be replaced with a simpler relation

$$
\partial_{t} \check{C}(k, t)+\alpha_{0} k^{2} \bar{G}(k, t)=0 \quad(\text { for } \forall k),
$$

in the sense that both sides of Eq. (4.5) vanishes if Eq. (4.7) holds, with Eq. (4.6) taken into account, of course. The constant $\alpha_{0}$ is determined to be $\alpha_{0}=D_{*} / L^{2}$ by the initial condition.

Taking notice of the property of Eq. (2.12) that the propagator $\bar{G}$ is equivalent to the response to the probe force, we note that Eq. (4.7) states the fluctuationdissipation theorem (FDT), which can be derived directly from the Langevin equation (2.12) through the distribution function [53, 80, 81]. In other words, FDT is already included in Eqs. (4.1) and (4.2). This inclusion is a remarkable feature of Eq. (2.12), or Eq. (2.8), if we compare it with an analogous calculation starting from the Fourier representation of the "Eulerian" equation (2.2), as opposed to the "Lagrangian" equation (2.8). In the "Eulerian" case, the step corresponding to the rearrangement of Eq. (4.5) turns out to be inconsistent with FDT [79]. This inconsistency is due to the hidden dependence of $f_{\rho}(x, t)$ on $\rho$ in its statistics in Eq. (2.3), known as the multiplicative noise [49], which makes the "Eulerian" equation intractable with a DIA-like expansion. Contrastively, the statistics of $f_{\mathrm{L}}$ on the right-hand side of the "Lagrangian" equation (2.8) is given by Eq. (2.9) which is independent of $\psi$. This is why the DIA equations (4.1) and (4.2) successfully reproduce FDT.

Equation (4.7) allows us to eliminate $\bar{G}$ from Eq. (4.1) and thereby elude the difficulties mentioned at the beginning of this subsection, as it implies

$$
\bar{G}(k, t)=-\frac{1}{\alpha_{0} k^{2}} \partial_{t} \check{C}(k, t),
$$

from which we can show

$$
M_{G}(k, s)=-\frac{1}{\alpha_{0} k^{2}} \partial_{s} M_{C}(k, s) .
$$

Then we substitute it into Eq. (4.1), and the result reads

$$
\begin{aligned}
& \left(\partial_{t}+\frac{D_{*}}{S} k^{2}\right) \check{C} \\
& =\frac{1}{\alpha_{0} k^{2}}\left[M_{C}(k, 0) \check{C}-\int_{0}^{t} \mathrm{~d} t^{\prime} M_{C}\left(k, t-t^{\prime}\right) \partial_{t^{\prime}} \check{C}\left(k, t^{\prime}\right)\right] .
\end{aligned}
$$

The source of the ultraviolet divergence is now isolated in $M_{C}(k, 0)$, which we should discard, as this term seems to have originated from an inappropriate treatment of the self-interaction in DIA [82]. Thus we arrive at the MCT equation:

$$
\left(\partial_{t}+\frac{D_{*}}{S} k^{2}\right) \check{C}(k, t)=-\int_{0}^{t} \mathrm{~d} t^{\prime} M\left(k, t-t^{\prime}\right) \partial_{t^{\prime}} \check{C}\left(k, t^{\prime}\right)
$$

where

$$
\begin{aligned}
M(k, s) & =\frac{M_{C}(k, s)}{\alpha_{0} k^{2}} \\
& =\frac{2 L^{4}}{N} D_{*} k^{2} \sum_{p+q+k=0} W_{p q k}^{2} \check{C}(p, s) \check{C}(q, s)
\end{aligned}
$$

with the summation taken over $(p, q)$ satisfying the triad condition $p+q+k=0$.

\section{Solution to MCT equation}

Now the finite-time correction to Eq. (3.3) for 2pDC is within our reach: all we need to do is to solve Eq. (4.8) and substitute the solution $\check{C}$ into the extended Alexander-Pincus formula (3.2). Although one may switch to numerical remedy, here we prefer to stick to the fully analytical calculation, which is possible by assuming the dilute limit $\left(\rho_{0} \sigma \rightarrow+0 ; S=1, D_{\mathrm{c}}=D\right)$. This does not trivialize the problem, because nonlinearity still exists due to

$$
\frac{1}{1+\psi}=1-\psi+\psi^{2}-\cdots
$$

and therefore the right-hand side of the MCT equation (4.8) does not vanish. Let us evaluate it using the linear solution Eq. (2.17) as the zeroth approximation valid for $t \rightarrow+\infty$, which now reads

$$
\check{C}(k, t) \simeq \frac{1}{L^{2}} e^{-D_{*} k^{2} t}
$$

as $S=1$.

To start with, we calculate $M(k, s)$ by substituting the approximate solution into Eq. (4.9). Parametrizing the variables in the summation as $(p, q)=(-k / 2+m,-k / 2-$ 
$m$ ) and denoting the wave number interval with $\Delta m=$ $2 \pi / N$, we find

$$
M(k, s)=\frac{D_{*} k^{2}}{\pi} \sum_{m} \exp \left[-D_{*}\left(\frac{1}{2} k^{2}+2 m^{2}\right) s\right] \Delta m .
$$

The summation is then replaced with an integral, which readily yields

$$
M(k, s)=\frac{D_{*} k^{2}}{\sqrt{2 \pi D_{*} s}} e^{-\frac{1}{2} D_{*} k^{2} s} .
$$

At this point, the nonlinear integro-differential equation, consisting of Eqs. (4.8) and (4.9), is approximated with a linear integro-differential equation that can be obtained by substituting Eq. (4.12) into Eq (4.8). The equation is then formally solved in terms of Laplace transform, but its inversion is difficult to perform analytically. Thus we need a further approximation: using both Eq. (4.11) and Eq. (4.12), we have

$$
\begin{aligned}
& \text { [RHS of Eq. (4.8)] } \\
& \qquad=\frac{D_{*}^{2} k^{4}}{L^{2}} e^{-D_{*} k^{2} t} \int_{0}^{t} \frac{\mathrm{d} t^{\prime}}{\sqrt{2 \pi D_{*}\left(t-t^{\prime}\right)}} e^{+\frac{1}{2} D_{*} k^{2}\left(t-t^{\prime}\right)},
\end{aligned}
$$

so that Eq. (4.8) is now approximated by a linear inhomogeneous differential equation.

Though the integral in Eq. (4.13) can be evaluated rigorously in terms of the error function with an imaginary argument, it is more convenient to evaluate it by expanding the integrand in powers of $t^{\prime}-t$, as the main contribution to the integral comes from the vicinity of $t^{\prime}=t$. Thus we find

[RHS of Eq. (4.8)]

$$
=\frac{D_{*} k^{4}}{L^{2}} e^{-D_{*} k^{2} t}\left[\sqrt{\frac{2}{\pi} D_{*} t}+\frac{k^{2}}{3} \sqrt{\frac{\left(D_{*} t\right)^{3}}{2 \pi}}+\cdots\right],
$$

which allows us to integrate Eq. (4.8) as

$$
\begin{aligned}
& \check{C}=\frac{1}{L^{2}} e^{-D_{*} k^{2} t} \times \\
& {\left[1+\frac{2}{3} \sqrt{\frac{2}{\pi}} k^{4}\left(D_{*} t\right)^{3 / 2}+\frac{2}{15 \sqrt{2 \pi}} k^{6}\left(D_{*} t\right)^{5 / 2}+\cdots\right] .}
\end{aligned}
$$

It should be possible, at least in principle, to substitute Eq. (4.14) into Eq. (4.9) and the right-hand side of Eq. (4.8) for the second approximation, but for the present let us content ourselves with this first approximation and go ahead.

However, some remarks on the properties of $W_{k p q}$ with finite $\rho_{0} \sigma$ may be in order here. At first glance,

$$
W_{k p q}=1+\frac{k}{p q} \sin \rho_{0} \sigma k+\frac{p}{k q} \sin \rho_{0} \sigma p+\frac{q}{k p} \sin \rho_{0} \sigma q
$$

might be reminiscent of the MCT vertex for fluids in disordered porous media [58, 83] and give an impression that it exhibits some singularity for $k \rightarrow 0$, but in actuality it does not. Under the condition that $k+p+q=0$, we have

$$
W_{k p q} \simeq 1+\rho_{0} \sigma \frac{k^{3}+p^{3}+q^{3}}{k p q}=1+3 \rho_{0} \sigma,
$$

which is evidently finite; the full treatment of the trigonometric functions in $W_{k p q}$ does not change the result. This behavior is parallel to that of the vertex for the corresponding Eulerian MCT. The one-dimensional Eulerian MCT for rigid particles with diameter $\sigma$ is given by Eq. (1.1) and

$$
M(k, s) \propto D k^{2} \sum\left(\frac{\sin \sigma p+\sin \sigma q}{k}\right)^{2} F(p, s) F(q, s) ;
$$

using $p+q+k=0$, we have $(\sin \sigma p+\sin \sigma q) / k \simeq-\sigma$ for the long-wave behavior of the vertex, which exhibits no singularity. Thus it is found that both the Lagrangian MCT vertex for $\check{C}$ and the Eulerian MCT vertex for $F$ are regular for long waves. The long-wave singularity responsible to the anomalous diffusion resides not in the memory kernel but in the modified Alexander-Pincus formula (2.15).

One may also wonder whether the Lagrangian MCT equation (4.8) exhibits an MCT transition and, if it occurs, what would be its consequence. A full study of the possible MCT transition, which means emergence of a non-trivial fixed point in the MCT dynamics, requires numerical evaluation of the wavenumber integral in Eq. (4.9) and therefore out of the scope of the present study. However, we may conjecture that the MCT transition would not affect the results of the present analysis seriously. Since the MSD given by the modified Alexander-Pincus formula (2.15) is dominated by the long-wave components of $\check{C}$, which is supposed to evolve very slowly, the behavior of $\left\langle R^{2}\right\rangle$ may remain essentially unchanged, at least within some limited time scale, even if an MCT transition occurs and $\check{C}(k, t)$ is destined to have some non-zero value for $t \rightarrow+\infty$. Numerical studies of Eq. (4.8) may clarify the validity range of this conjecture and will be reported elsewhere.

\section{Effects of the nonlinear term on transient behaviors of MSD and 2pDC}

Since $\check{C}$ is now available in Eq. (4.14) as a result of nonlinear closure theory, we can evaluate $\left\langle R(\xi, t) R\left(\xi^{\prime}, t\right)\right\rangle$ using the formula (3.2). The procedure is analogous to that for the derivation of Eq. (3.3) from the linear solution in Eq. (2.17).

If we take into account the term of order $\left(D_{*} t\right)^{3 / 2}$ and ignore that of order $\left(D_{*} t\right)^{5 / 2}$ in Eq. (4.14), by substituting Eq. (4.14) into the formula (3.2) we obtain 


$$
\begin{aligned}
\left\langle R(\xi, t) R\left(\xi^{\prime}, t\right)\right\rangle & =\frac{2}{\rho_{0}} \sqrt{\frac{D t}{\pi}} \exp \left[-\frac{\left(\xi-\xi^{\prime}\right)^{2}}{4 \rho_{0}^{2} D t}\right]-\frac{\left|\xi-\xi^{\prime}\right|}{\rho_{0}^{2}} \operatorname{erfc} \frac{\left|\xi-\xi^{\prime}\right|}{2 \rho_{0} \sqrt{D t}}-\frac{\sqrt{2}}{3 \pi} \rho_{0}^{-2}\left[1-\frac{\left(\xi-\xi^{\prime}\right)^{2}}{2 \rho_{0}^{2} D t}\right] \exp \left[-\frac{\left(\xi-\xi^{\prime}\right)^{2}}{4 \rho_{0}^{2} D t}\right] \\
& =\sigma \sqrt{D t} \varphi(\theta)-\frac{\sqrt{2}}{3 \pi} \rho_{0}^{-2}\left(1-2 \theta^{2}\right) e^{-\theta^{2}}
\end{aligned}
$$

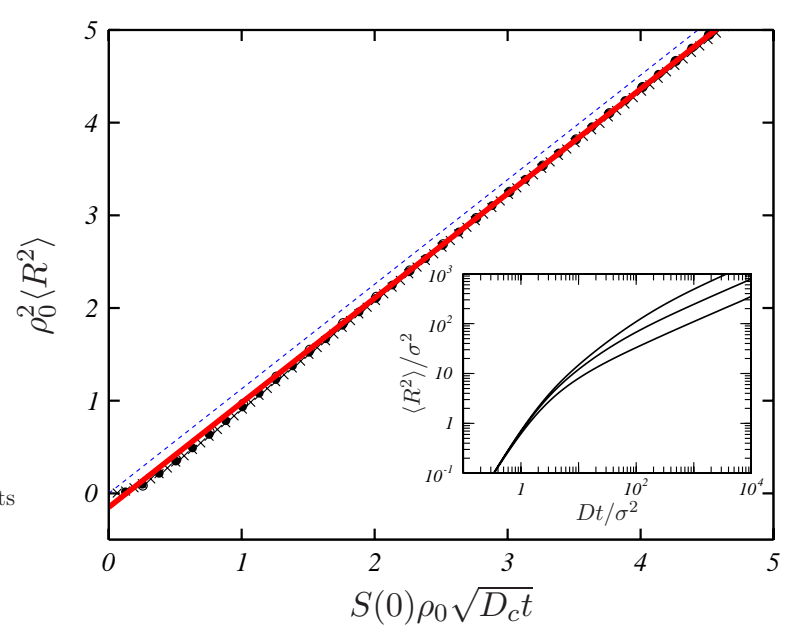

FIG. 5: (Color online) Comparison of Eq. (4.16) with numerical data, by means of rescaled plotting of MSD versus time for three different values of the density: $\rho_{0}=N / L=(1 / 4) \sigma^{-1}$, $(1 / 8) \sigma^{-1}$, and $(1 / 16) \sigma^{-1}$. The number of the particles is fixed at $N=2^{15}=32768$. The solid (red) line represents the prediction of the nonlinear theory in Eq. (4.16), which is compared with Kollmann's law (1.4) in the dotted line. See the text for the reason why $S(0)$ is included in the rescaling. The inset shows the same data $\left(\rho_{0} \sigma=1 / 4,1 / 8\right.$, and $1 / 16$, from bottom to top) without rescaling, using the simple nondimensionalization with $\sigma^{2}$ and $\sigma^{2} / D$.

where $\theta=\left(\xi-\xi^{\prime}\right) /\left(2 \rho_{0} \sqrt{D t}\right)$ and the function $\varphi$ is defined in Eq. (3.3) with $S=1$. As a special case for $\xi=\xi^{\prime}$, Eq. (4.15) gives correction to $\left\langle R^{2}\right\rangle \propto \sqrt{t}$ :

$$
\left\langle R^{2}\right\rangle=\frac{2}{\rho_{0}} \sqrt{\frac{D t}{\pi}}-\frac{\sqrt{2}}{3 \pi} \rho_{0}^{-2} .
$$

The first term reproduces Eq. (1.4) with $S=1$, while the second term gives a correction to it. The contribution from the higher-order terms in Eq. (4.14) slightly enlarges the coefficient of the correction term, but the form of Eq. (4.16) itself is not affected. It is interesting to note that Eq. (4.16), if combined with the relation [24, 84]

$$
\frac{\mathrm{d}^{2}\left\langle R^{2}\right\rangle}{\mathrm{d} t^{2}}=2\langle u(t) u(0)\rangle
$$

with $u=\mathrm{d} R / \mathrm{d} t$, gives the same expression as the asymptotic one without the correction term.

While the first term in Eq. (4.16) is a mere reconfirmation of the classical result, the second term is something non-trivial which should be verified numerically. Without the second term in Eq. (4.16), plotting $\left\langle R^{2}\right\rangle$ against $\sqrt{t}$ would yield a graph of a straight line passing through the origin. In actuality, the second term in Eq. (4.16) shifts the asymptotic straight line, making a positive intercept on the $\sqrt{t}$-axis and a negative intercept on the $\left\langle R^{2}\right\rangle$-axis. These intercepts are already visible in the inset of Fig. 1 and also in Fig. 5 (a plot analogous to Fig. 1 but with the axes rescaled, as we explain below). The presence of the correction itself is probably not surprising, because the short-time diffusion should behave as $\left\langle R^{2}\right\rangle \propto D t$ before the collisions begin to take effect; it is more noteworthy that, since this single-particle behavior plays the role of the "mode-coupling" in the Fourier representation, the description of the transient behavior requires a nonlinear theory such as MCT. Viewed in the light of the thermodynamic potential, the nonlinearity in Eq. (4.10) originates from that of the configurational entropy. This nonlinearity is captured by adopting $\psi(\xi, t)$ as the field variable, which could be interpreted as a kind of kinetic free-volume theory.

The prediction of the nonlinear theory in Eq. (4.16), including its dependence on the density $\rho_{0}$, is compared with the computed MSD in Fig. 5 Having noticed that the quantitative comparison requires us to take into account the effects of the finite density, we revived $S=S(0)$ in the first term of Eq. (4.16), and plotted $\rho_{0}^{2}\left\langle R^{2}\right\rangle$ against $S \rho_{0} \sqrt{D_{\mathrm{c}} t}$. The MSD computed for three different values of density $\left(\rho_{0} \sigma=1 / 4,1 / 8\right.$, and $1 / 16$; see the inset) seem to collapse into a single curve whose asymptote is the straight line given by Eq. (4.16). Improvement of Eq. (4.16) accounting for the small deviation from the straight line, as well as justification for the revival of $S$ in Eq. (4.16), will be performable with a careful numerical calculation of the MCT equation, which will be reported elsewhere.

\section{OTHER FORMS OF FOUR-POINT CORRELATION DERIVED FROM 2PDC}

\section{A. Behavior of $2 \mathrm{pDC}$ as a function of the initial distance}

As we have already shown in Fig. 4, the theoretical prediction for $\left\langle R(\xi, t) R\left(\xi^{\prime}, t\right)\right\rangle$ is almost perfectly consistent with numerical calculation. However, taking the label distance $\xi-\xi^{\prime}$ or $\theta=\left(\xi-\xi^{\prime}\right) /\left[\rho_{0} \lambda(t)\right]$ for the horizontal axis of the graph is sometimes inconvenient, for example, when we try to compare the result with two-dimensional or three-dimensional numerical calculations. For this purpose, it may be more convenient to 


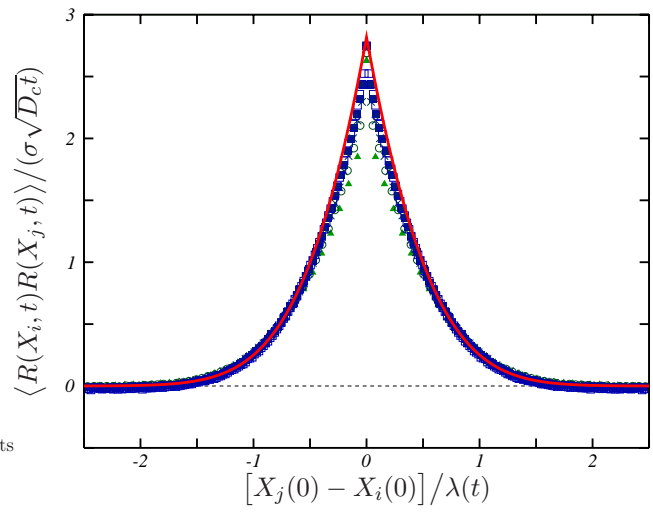

FIG. 6: (Color online) Eq. (3.3) compared with simulation data (with the same $N$ and $\rho_{0}$ as in Figs. 1 and 4), on the basis of the initial distance $X_{j}(0)-X_{i}(0)$ instead of the particle numbering. The solid (red) line shows the theoretical prediction for $t \rightarrow+\infty$ by Eq. (3.3), while the simulation data are plotted with the same symbols as in Fig. 4 .

re-express the result as a function of the initial distance, $X_{j}(0)-X_{i}(0)$, and plot the two-particle displacement correlation against $\left[X_{j}(0)-X_{i}(0)\right] / \lambda(t)$. Such a graph is shown in Fig. 6. The analytic curve in Fig. 6 is drawn by simply equating $\left[X_{j}(0)-X_{i}(0)\right] / \lambda(t)$ with $\theta$ in Eq. (3.3]). This seems to be valid for $t \rightarrow+\infty$, but a considerable discrepancy is seen for shorter times.

Although $X_{j}(0)-X_{i}(0)$ and $\left(\Xi_{j}-\Xi_{i}\right) / \rho_{0}$ are equal on the average, generally they are different, as is evident from the relation

$$
X_{j}(0)-X_{i}(0)=\int_{\Xi_{i}}^{\Xi_{j}} \frac{1+\psi(\xi, 0)}{\rho_{0}} \mathrm{~d} \xi .
$$

This difference is responsible for the discrepancy in Fig. 6 for finite $t$. Taking this difference into account, we can evaluate $2 \mathrm{pDC}$ theoretically as a function of the initial distance. Although its complete evaluation is out of the scope of the present paper, as it seems to involve wavenumber integrals that are difficult to perform analytically, we can outline the procedure of the calculation at least.

With the value of the initial distance denoted with $\tilde{d}$, the function that gives $2 \mathrm{pDC}$, which we denote with $X_{R}(\tilde{d}, t)$, is formally written as

$$
\begin{aligned}
& X_{R}(\tilde{d}, t) \\
& \quad=\left\langle\frac{1}{L} \iint \delta\left(x_{1}-x_{2}-\tilde{d}\right) R\left(\xi_{1}, t\right) R\left(\xi_{2}, t\right) \mathrm{d} x_{1} \mathrm{~d} x_{2}\right\rangle
\end{aligned}
$$

where

$$
x_{1}=x_{1}\left(\xi_{1}\right)=x\left(\xi_{1}, 0\right), \quad x_{2}=x_{2}\left(\xi_{2}\right)=x\left(\xi_{2}, 0\right),
$$

and therefore

$$
x_{2}-x_{1}=\frac{1}{\rho_{0}}\left[\xi_{2}-\xi_{1}+\int_{\xi_{1}}^{\xi_{2}} \psi(\xi, 0) \mathrm{d} \xi\right] .
$$

Changing the variables of the integral in Eq. (5.2) from $\left(x_{1}, x_{2}\right)$ to $\left(\xi_{1}, \xi_{2}\right)$ with

$$
\mathrm{d} x_{1}=\frac{1+\psi\left(\xi_{1}, 0\right)}{\rho_{0}} \mathrm{~d} \xi_{1}, \quad \mathrm{~d} x_{2}=\frac{1+\psi\left(\xi_{2}, 0\right)}{\rho_{0}} \mathrm{~d} \xi_{2},
$$

and introducing the Fourier representation of the delta function,

$$
\delta(\tilde{x})=\frac{1}{L} \sum_{q} e^{-\mathrm{i} q \tilde{x}} \quad\left(\frac{q}{2 \pi / L} \in \mathbb{Z}\right),
$$

we rewrite Eq. (5.2) as

$$
X_{R}(\tilde{d}, t)=\sum_{k} e^{\mathrm{i} k \rho_{0} \tilde{d}}\langle\tilde{R}(k, t) \tilde{R}(-k, t)\rangle
$$

where $k=q / \rho_{0}$ and

$$
\begin{aligned}
\tilde{R}(k, t) & =\frac{1}{L} \int_{0}^{L} e^{\mathrm{i} q x} R(\xi(x, 0), t) \mathrm{d} x \\
& =\frac{1}{N} \int_{0}^{N} \exp \left(\mathrm{i} k\left[\xi+\int_{0} \psi \mathrm{d} \xi\right]\right) R(\xi, t)(1+\psi) \mathrm{d} \xi
\end{aligned}
$$

with

$$
\psi=\psi(\xi, 0), \quad \int_{0} \psi \mathrm{d} \xi=\int_{0}^{\xi} \psi(\tilde{\xi}, 0) \mathrm{d} \tilde{\xi}
$$

Then we express $\psi$ in Eq. (5.4) with $\check{\psi}$ in Eq. (2.11) and also substitute Eq. (3.1). After some rearrangement, we obtain

$$
\begin{aligned}
& \tilde{R}(k, t)=\frac{L}{N} \times \frac{\check{\psi}(k, t)-\check{\psi}(k, 0)}{-\mathrm{i} k} \\
& +\frac{L}{N} \sum_{p+p^{\prime}=k} \frac{\check{\psi}(p, 0)\left[\check{\psi}\left(p^{\prime}, t\right)-\check{\psi}\left(p^{\prime}, 0\right)\right]}{\mathrm{i} p}+O\left(\check{\psi}^{3}\right) .
\end{aligned}
$$

Substituting Eq. (5.5) into Eq. (5.3) yields an expression of $X_{R}(\tilde{d}, t)$ that consists of two parts: the first part simply reproduces Eq. (3.2) with $\xi-\xi^{\prime}$ replaced with $\rho_{0} \tilde{d}$, and the second part involves triple correlations such as $\langle\breve{\psi}(-p, 0) \check{\psi}(-q, t) \check{\psi}(-k, t)\rangle$ with $p+q+k=0$. These triple correlations can be calculated with DIA, and as a result, we obtain a correction term whose magnitude relative to the leading term decreases in proportion to $t^{-1 / 2}$ for $t \rightarrow+\infty$. Detailed results of the calculation will be reported elsewhere.

We note that the definition of $X_{R}$ in Eq. (5.2) is readily generalized to three-dimensional cases, as

$$
\begin{aligned}
\mathbf{X}(\tilde{\mathbf{d}}, t) & =\left\langle\frac{1}{L^{3}} \iint \delta^{3}\left(\mathbf{r}_{12}-\tilde{\mathbf{d}}\right) \mathbf{R}_{1} \otimes \mathbf{R}_{2} \mathrm{~d}^{3} \mathbf{r}_{1} \mathrm{~d}^{3} \mathbf{r}_{2}\right\rangle \\
& =\left\langle\frac{L^{3}}{N^{2}} \sum_{i} \sum_{j} \frac{\delta^{3}\left(\mathbf{r}_{i j}-\tilde{\mathbf{d}}\right)}{g_{2}\left(\mathbf{r}_{i j}\right)} \mathbf{R}_{i} \otimes \mathbf{R}_{j}\right\rangle
\end{aligned}
$$


where $\mathbf{r}_{i j}=\mathbf{r}_{j}-\mathbf{r}_{i}, \mathbf{R}_{i}=\mathbf{R}\left(\boldsymbol{\xi}_{i}, t\right)=\mathbf{r}_{i}(t)-\mathbf{r}_{i}(0)$, and

$$
g_{2}(\mathbf{r})=\frac{L^{3}}{N^{2}} \sum_{i^{\prime}} \sum_{j^{\prime}} \delta^{3}\left(\mathbf{r}_{j^{\prime}}-\mathbf{r}_{i^{\prime}}-\mathbf{r}\right) .
$$

This $\mathrm{X}$ is similar to the quantity calculated by Donati et al. 44] ( $g_{u}$ in their notation), except for two main differences: in their $g_{u}$, the two-body density $g_{2}$ is absent in the denominator, and a product of scalar displacements, $\left|\mathbf{R}_{i}\right|\left|\mathbf{R}_{j}\right|$, is used instead of the tensor product $\mathbf{R}_{i} \otimes \mathbf{R}_{j}$. The presence or absence of $g_{2}$ is not essential, though it indeed makes it difficult to define $X$ for small values of the initial distance, $|\tilde{\mathbf{d}}|<\sigma$, in which we are not interested. The other difference is crucial: the absolute value signs obstruct analytical evaluation of $g_{u}$ even in the one-dimensional cases. Besides, the tensorial character of $\mathrm{X}$ can provide useful information on the geometry of the collective motion in the three-dimensional glassy systems. We will return to this point in Sec. VI, but before that, let us relate the one-dimensional $2 \mathrm{pDC}$ to $\chi_{4}$.

\section{B. Calculation of $\chi_{4}$ from $2 \mathrm{pDC}$}

With the knowledge of the displacement correlation $\left\langle R(\xi, t) R\left(\xi^{\prime}, t\right)\right\rangle$ in Eq. (4.15), we can also calculate a one-dimensional version of a quantity which is commonly referred to as $\chi_{4}(t)$. To be precise, we consider the $\mathcal{Q}$ based $\chi_{4}$ [85, 86], as opposed to other variants of $\chi_{4}$ such as the $F$-based $\chi_{4}$ [18, 87] defined through the fluctuation of the intermediate scattering function $F$ or its self part. If we consult Glotzer et al. 85. and adapt their equations (4) and (5) for one-dimensional cases, we have

$$
\begin{gathered}
\mathcal{Q}=\sum_{i} \sum_{j} \bar{\delta}_{a}\left(X_{j}(t)-X_{i}(0)\right), \\
\chi_{4}(t)=\frac{L}{k_{\mathrm{B}} T} \frac{\left\langle\mathcal{Q}^{2}\right\rangle-\langle\mathcal{Q}\rangle^{2}}{N^{2}},
\end{gathered}
$$

with some radius $a$ as a criterion of overlapping; $\bar{\delta}_{a}$ denotes the overlapping function, which has a finite value around $r=0$ and vanishes for $r \gg a$.

This type of four-point correlation function has been studied by many authors [85, 86, 88 91] as an indicator of cooperative motion in glassy systems. To our knowledge, most of these studies are based on direct numerical simulations of particle systems and there are also experiments grounded on observation of particles, but analytical calculations are quite rare. What makes it difficult to calculate $\chi_{4}$ analytically is that, in the usual formulation, the four-point correlation implies a four-body correlation. More concretely, as $\mathcal{Q}$ in Eq. (5.7) already contains double summation, calculation of $\chi_{4}$ requires dealing with quadruple summation whose summand involves four particles simultaneously; this would be a hopeless task.

To facilitate calculation of four-point correlation, here we introduce two modifications to Eqs. (5.7) and (5.8). Firstly, we target on the "self part" $(i=j)$ of $\mathcal{Q}$ and its contribution to $\chi_{4}$, denoting them as $[92$ ]

$$
\mathcal{Q}_{\mathrm{S}}=\sum_{i} \bar{\delta}_{a}\left(R_{i}(t)\right), \quad \chi_{4}^{\mathrm{S}}(t)=\frac{L}{k_{\mathrm{B}} T} \frac{\left\langle\mathcal{Q}_{\mathrm{S}}^{2}\right\rangle-\left\langle\mathcal{Q}_{\mathrm{S}}\right\rangle^{2}}{N^{2}} .
$$

Since Glotzer et al. [85] reported that the contribution of the self part $(i=j)$ is dominant over that of the distinct part $(i \neq j)$ in the three-dimensional case, it is justifiable to calculate $\chi_{4}^{\mathrm{S}}$ instead of $\chi_{4}$. Secondly, as the overlap function $\bar{\delta}_{a}$, we adopt a Gaussian function

$$
\bar{\delta}_{a}(r)=e^{-r^{2} / a^{2}}
$$

instead of the step function used by Glotzer et al. [85]. We note that, although there exists a variant of $\chi_{4}$ from whose definition the probe length can be totally expelled [71], the probe length $a$ is indispensable to $\mathcal{Q}_{\mathrm{S}}$.

From Eq. (4.15) we already know the covariance $\left\langle R_{i} R_{j}\right\rangle$ for all $(i, j)$ and for arbitrary $t$ (within a certain limitation, of course). The problem is how to evaluate $\left\langle\mathcal{Q}_{\mathrm{S}}\right\rangle$ and $\left\langle\mathcal{Q}_{\mathrm{S}}^{2}\right\rangle$ in Eq. (5.9) using this information. This is possible, if we assume that $\left(R_{1}, R_{2}, \ldots, R_{N}\right)$ is subject to a joint (multivariate) Gaussian distribution, which is determined uniquely as the covariance is given and the mean is known to vanish. For the purpose of calculating $\left\langle\mathcal{Q}_{\mathrm{S}}^{2}\right\rangle$, it suffices to determine the two-body distribution function for $\left(R_{i}, R_{j}\right)$, which we denote with

$$
\begin{aligned}
& P\left(R_{i}, R_{j}\right) \\
= & \frac{1}{2 \pi \sqrt{\Delta_{i j}}} \exp \left[-\frac{\left\langle R^{2}\right\rangle\left(R_{i}^{2}+R_{j}^{2}\right)-2\left\langle R_{i} R_{j}\right\rangle R_{i} R_{j}}{2 \Delta_{i j}}\right]
\end{aligned}
$$

where

$$
\Delta_{i j}=\left\langle R^{2}\right\rangle^{2}-\left\langle R_{i} R_{j}\right\rangle^{2}, \quad\left\langle R^{2}\right\rangle=\left\langle R_{i}^{2}\right\rangle=\left\langle R_{j}^{2}\right\rangle
$$

Using this joint distribution function $P\left(R_{i}, R_{j}\right)$ and adopting Eq. (5.10) for the overlapping function, we obtain

$$
\begin{aligned}
\left\langle\bar{\delta}_{a}\left(R_{i}\right)\right\rangle & =\int \bar{\delta}_{a}\left(R_{i}\right) P\left(R_{i}, R_{j}\right) \mathrm{d} R_{i} \mathrm{~d} R_{j} \\
& =\frac{1}{\sqrt{1+\frac{2\left\langle R^{2}\right\rangle}{a^{2}}}}
\end{aligned}
$$

and

$$
\begin{aligned}
\left\langle\bar{\delta}_{a}\left(R_{i}\right) \bar{\delta}_{a}\left(R_{j}\right)\right\rangle & =\int \bar{\delta}_{a}\left(R_{i}\right) \bar{\delta}_{a}\left(R_{j}\right) P\left(R_{i}, R_{j}\right) \mathrm{d} R_{i} \mathrm{~d} R_{j} \\
& =\frac{1}{\sqrt{\left(1+\frac{2\left\langle R^{2}\right\rangle}{a^{2}}\right)^{2}-\frac{4\left\langle R_{i} R_{j}\right\rangle^{2}}{a^{4}}}}
\end{aligned}
$$

note that Eq. (5.12) is confirmed separately for $i \neq j$ and $i=j$. With Eq. (5.11) and (5.12), now we can evaluate $\chi_{4}^{\mathrm{S}}$ in Eq. (5.9), taking the uniformity of the system into account. As a result, we obtain 


$$
\begin{aligned}
\chi_{4}^{\mathrm{S}} & =\frac{L}{N^{2} k_{\mathrm{B}} T}\left\{\sum_{i} \sum_{j}\left\langle\bar{\delta}_{a}\left(R_{i}\right) \bar{\delta}_{a}\left(R_{j}\right)\right\rangle-\left[\sum_{i}\left\langle\bar{\delta}_{a}\left(R_{i}\right)\right\rangle\right]^{2}\right\} \\
& =\frac{L}{N k_{\mathrm{B}} T} \sum_{l}\left[\frac{1}{\sqrt{\left(1+\frac{2\left\langle R^{2}\right\rangle}{a^{2}}\right)^{2}-\frac{4\left\langle R_{i} R_{i+l}\right\rangle^{2}}{a^{4}}}}-\frac{1}{1+\frac{2\left\langle R^{2}\right\rangle}{a^{2}}}\right] .
\end{aligned}
$$

Note that the double summation $\sum_{i} \sum_{j}\left\langle\bar{\delta}_{a}\left(R_{i}\right) \bar{\delta}_{a}\left(R_{j}\right)\right\rangle$ in Eq. (5.13) is a result of the simplification by the replacement of $\chi_{4}$ with $\chi_{4}^{\mathrm{S}}$ (retaining only the self part): if this simplification were not introduced, we would have to struggle with a quadruple summation such as

$$
\sum_{i} \sum_{j} \sum_{k} \sum_{l}\left\langle\bar{\delta}_{a}\left(X_{j}(t)-X_{i}(0)\right) \bar{\delta}_{a}\left(X_{l}(t)-X_{k}(0)\right)\right\rangle,
$$

whose evaluation would be much less workable than $\left\langle\bar{\delta}_{a}\left(R_{i}\right) \bar{\delta}_{a}\left(R_{j}\right)\right\rangle$.

Before applying Eq. (5.13) to SFD, we can test it with free Brownian particles. From the Langevin equation obtained by setting $V=0$ in Eq. (1.2), we have

$$
\left\langle R_{i} R_{j}\right\rangle= \begin{cases}\left\langle R^{2}\right\rangle=2 D\left[t-\tau_{\mathrm{B}}\left(1-e^{-t / \tau_{\mathrm{B}}}\right)\right] & (i=j) \\ 0 & (i \neq j)\end{cases}
$$

where $\tau_{\mathrm{B}}=m / \mu$. This is substituted into Eq. (5.13), which yields

$$
\begin{aligned}
\chi_{4}^{\mathrm{S}} & =\frac{1}{\rho_{0} k_{\mathrm{B}} T}\left(\frac{1}{\sqrt{1+4\left\langle R^{2}\right\rangle / a^{2}}}-\frac{1}{1+2\left\langle R^{2}\right\rangle / a^{2}}\right) \\
& =\left(\chi_{4}^{\mathrm{S}}\right)_{\text {solo }}
\end{aligned}
$$

for free Brownian particles; note that all the contribution comes from the term with $l=0$ in Eq. (5.13), which we refer to as the "solo" part. Taking notice of the $t$ dependence of $\left\langle R^{2}\right\rangle$ in Eq. (5.15) and making some calculation, we find $\left(\chi_{4}^{\mathrm{S}}\right)_{\text {solo }}$ to have a peak at the instant when $\left\langle R^{2}\right\rangle=2.6 a^{2}$ approximately; see the short-time side of Fig. 7(a). Obviously, this short-time peak is irrelevant to particle interaction. After this peak, $\left(\chi_{4}^{S}\right)_{\text {solo }}$ decreases monotonically toward zero, in proportion to $t^{-1 / 2}$ for $t \rightarrow+\infty$.

Now let us calculate $\chi_{4}^{\mathrm{S}}$ for SFD, combining Eq. (5.13) with the result for $\langle R R\rangle$ in Eq. (4.15). We evaluate $\chi_{4}^{\mathrm{S}}$ in Eq. (5.13) as a sum of the "solo" part $(l=0)$ and the collective part (contribution from the terms with $l \neq 0$ ). The solo part is given by Eq. (5.15) and depends on $\left\langle R^{2}\right\rangle$ alone, for which we use Eq. (4.16) that was obtained by setting $\xi=\xi^{\prime}$ in Eq. (4.15). As a matter of course, we must exclude cases of very short time, for which Eq. (4.16) predicts $\left\langle R^{2}\right\rangle$ to be negative; this is out of the validity range of Eq. (4.16). Subsequently, to evaluate the contribution from the terms with $l \neq 0$, we use the asymptotic form of Eq. (4.15) for $t \rightarrow+\infty$, expressed as a self-similar solution in Eq. (3.31):

$$
\left\langle R_{i} R_{i+l}\right\rangle \simeq \frac{2 \sqrt{D t}}{\rho_{0}} \varphi\left(\theta_{l}\right), \quad \theta_{l}=\frac{l}{\rho_{0} \lambda(t)}=l \Delta \theta .
$$

The collective part is thereby written as

$$
\begin{aligned}
& \left(\chi_{4}^{\mathrm{S}}\right)_{\text {coll }} \\
& \simeq \frac{1}{\rho_{0} k_{\mathrm{B}} T} \times \frac{1}{1+\frac{2\left\langle R^{2}\right\rangle}{a^{2}}} \sum_{l \neq 0}\left\{\frac{1}{\sqrt{1-\left[\frac{\varphi\left(\theta_{l}\right)}{\varphi(0)}\right]^{2}}}-1\right\} .
\end{aligned}
$$

The number of particles contributing to the sum is estimated to be $N_{\text {coll }} \sim 1 / \Delta \theta=\rho_{0} \lambda(t)$, which yields, approximately,

$$
\begin{aligned}
\left(\chi_{4}^{\mathrm{S}}\right)_{\mathrm{coll}} & \sim \frac{1}{\rho_{0} k_{\mathrm{B}} T} \times \frac{\rho_{0} \lambda(t)}{1+2\left\langle R^{2}\right\rangle / a^{2}} \\
& \sim \frac{a^{2}}{k_{\mathrm{B}} T} \times \frac{\lambda(t)}{\left\langle R^{2}\right\rangle} \sim \frac{a^{2} \rho_{0}}{k_{\mathrm{B}} T}
\end{aligned}
$$

for $t \rightarrow+\infty$. More precisely, the sum can be evaluated by rewriting it as

$$
\begin{aligned}
\sum_{l \neq 0}(\cdots) & =2 \rho_{0} \lambda(t) \sum_{l=1}^{\infty}\left\{\frac{1}{\sqrt{1-\left[\frac{\varphi\left(\theta_{l}\right)}{\varphi(0)}\right]^{2}}}-1\right\} \Delta \theta \\
& \rightarrow 2 \rho_{0} \lambda(t) \int_{0}^{\infty}\left\{\frac{1}{\sqrt{1-\pi[\varphi(\theta)]^{2}}}-1\right\} \mathrm{d} \theta
\end{aligned}
$$

and using the numerical value of the integral

$$
\int_{0}^{\infty}\left\{\frac{1}{\sqrt{1-\pi[\varphi(\theta)]^{2}}}-1\right\} \mathrm{d} \theta=0.364124
$$


(a)

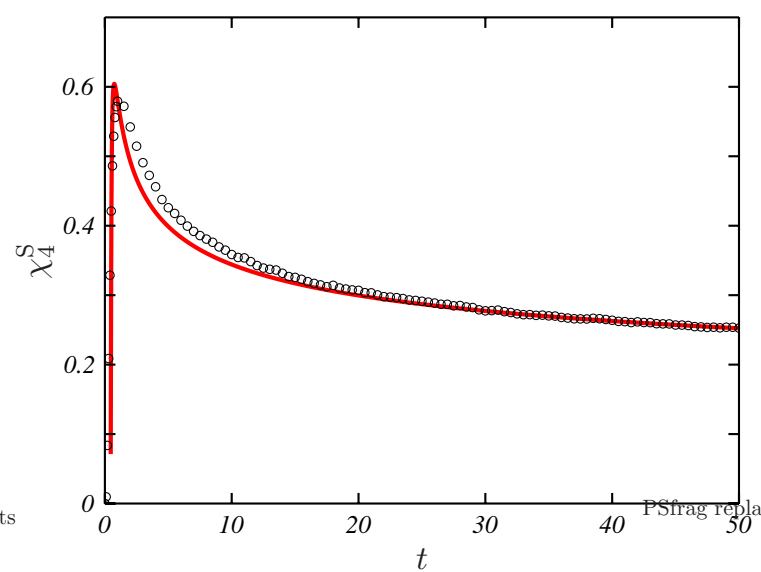

(b)

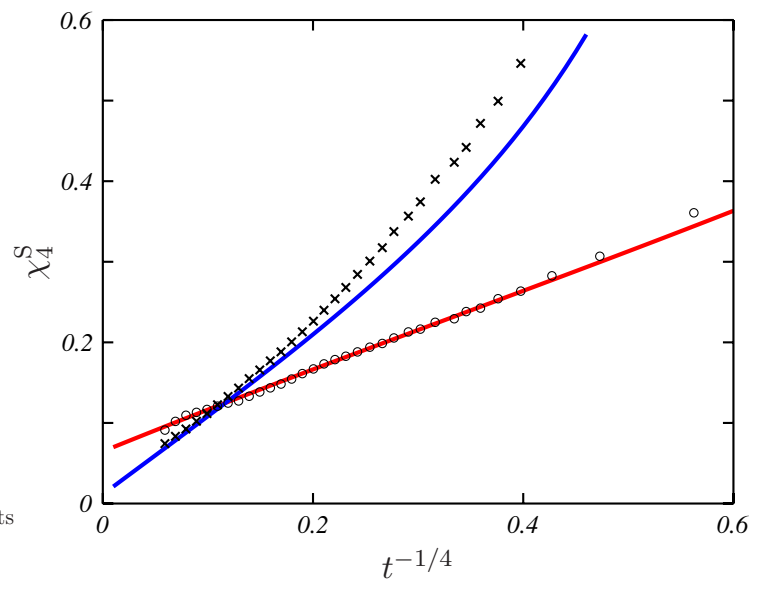

FIG. 7: (Color online) Short-time and long-time behavior of $\chi_{4}^{\mathrm{S}}$ for SFD, compared with the analytical prediction given by the sum of $\left(\chi_{4}^{\mathrm{S}}\right)_{\text {solo }}$ in Eq. (5.15) and $\left(\chi_{4}^{\mathrm{S}}\right)_{\text {coll }}$ in Eq. (5.17), with the effect of $S \neq 1$ and $D_{\text {c }} / D \neq 1$ taken into account. The probe length (radius of the overlapping function) was chosen as $a=0.5 \sigma$. The time is non-dimensionalized with $\sigma^{2} / D$. (a) Short-time behavior for the case with $N=256$ and $\rho_{0}=N / L=(1 / 4) \sigma^{-1}$. The computed data are plotted with circles and the analytical prediction is shown with a solid (red) line. (b) Long-time behavior for $\rho_{0}=(1 / 4) \sigma^{-1}$ (plotted with circles) and for $\rho_{0}=(1 / 16) \sigma^{-1}$ (plotted with crosses). The solid lines show analytical prediction for the two cases. Note the agreement between the computed results and the theoretical curves except for the transient discrepancy, as well as the asymptotic behavior of the curves that takes the form of a straight line in the graph, exhibiting the decay of $\left(\chi_{4}^{\mathrm{S}}\right)_{\text {solo }}$ in proportion to $t^{-1 / 4}$ and the finite value of $\left(\chi_{4}^{\mathrm{S}}\right)_{\text {coll }}$ that remains for $t \rightarrow+\infty$.

thus we obtain

$$
\begin{aligned}
\left(\chi_{4}^{\mathrm{S}}\right)_{\mathrm{coll}} & =\frac{1}{\rho_{0} k_{\mathrm{B}} T} \times \frac{\rho_{0} \lambda(t)}{\left\langle R^{2}\right\rangle / a^{2}} \times 0.364124 \\
& =\frac{1}{\rho_{0} k_{\mathrm{B}} T} \times \sqrt{\pi} \rho_{0}^{2} a^{2} \times 0.364124 \\
& =0.6454 \times \frac{\rho_{0} a^{2}}{k_{\mathrm{B}} T}
\end{aligned}
$$

and $\chi_{4}^{\mathrm{S}}=\left(\chi_{4}^{\mathrm{S}}\right)_{\text {solo }}+\left(\chi_{4}^{\mathrm{S}}\right)_{\text {coll }}$, with $\left(\chi_{4}^{\mathrm{S}}\right)_{\text {solo }}$ given by substituting Eq. (4.16) into Eq. (5.15). If we take $S$ into account according to Eq. (3.3), with the reservation that both Eq. (3.3]) and Eq. (5.17) are valid only asymptotically, the right-hand side of Eq. (5.17) is multiplied by $S^{-1}$.

The analytically calculated $\chi_{4}^{\mathrm{S}}$ and its numerical values are compared in Fig. 7. The effect of finite $\rho_{0}$ that makes $S$ and $D_{\text {c }} / D$ different from unity is taken into account (see Table 1 in Appendix A). The peak in the short-time regime has nothing to do with the slow dynamics, as it appears even for free Brownian particles. After this peak, $\left(\chi_{4}^{\mathrm{S}}\right)_{\text {solo }}$ decreases slowly, asymptotically in proportion to $t^{-1 / 4}$, while, reflecting the endless growth of the cluster size, $\left(\chi_{4}^{\mathrm{S}}\right)_{\text {coll }}$ remains finite for $t \rightarrow+\infty$. The behavior of the numerical solution is consistent with this analytical prediction.

The limiting value of $\chi_{4}^{\mathrm{S}}$ for $t \rightarrow+\infty$, given by $\left(\chi_{4}^{\mathrm{S}}\right)_{\text {coll }}$ in Eq. (5.17), is an increasing function of the density $\rho_{0}$. This conclusion remains unchanged also if the effect of $S \neq 1$ is included, because $1 / S$ is also an increasing function of $\rho_{0}$. In conformity with the theoretical prediction, Fig. [7(b) shows that the value of $\chi_{4}^{\mathrm{S}}$ is greater for $\rho_{0}=(1 / 4) \sigma^{-1}$ (circles) than for $\rho_{0}=(1 / 16) \sigma^{-1}$ (crosses) if $t$ is sufficiently long.

\section{DISCUSSION}

\section{A. Quantification of collective dynamics}

We have studied a one-dimensional system of Brownian particles with repulsive interaction, regarding it as a simplified model of the cage effect. On one hand, the cage confines every particle in a narrow space. On the other hand, since the particles are mutually caged and therefore forbidden to move uncooperatively, they must either wait still or move together. Thus the cage effect involves correlated motion of numerous particles, which is visualized as dynamical clusters in space-time diagrams (Fig. 3), with the diffusive correlation length $\lambda(t)=2 \sqrt{D_{\mathrm{c}} t}$. The substance that diffuses is not the particles but the space between them, represented by $\psi$ in our theory. The two-particle displacement correlation $(2 \mathrm{pDC})$ is shown to be a useful indicator of the correlated motion. Asymptotically, 2pDC becomes self-similar: it suggests a matryoshka-like structure, such that the small cages are confined in larger cages, which, in turn, are caught in still larger and slower cages.

Using the analytical result for $2 \mathrm{pDC}$ which is valid both transiently and asymptotically, we demonstrated how to calculate the $\mathcal{Q}_{\mathrm{S}}$-based $\chi_{4}$ (denoted with $\chi_{4}^{\mathrm{S}}$ ). Despite the endless growth of $\lambda(t)$ and the absence of $\alpha$ relaxation, the result in Eq. (5.17) shows that $\chi_{4}^{\mathrm{S}}$ converges to some constant for $t \rightarrow+\infty$. An implication of Eq. (5.17) is that $\chi_{4}^{\mathrm{S}}$, and probably $\chi_{4}$ in general, does 
not give a straightforward representation of the cluster size. Indeed, $\lambda(t)$ is in the numerator of Eq. (5.16) or (5.17), but the result is modified by the denominator, or a prefactor $1 /\left(1+2\left\langle R^{2}\right\rangle / a^{2}\right)$ originating from $\left\langle\mathcal{Q}_{\mathrm{S}}\right\rangle^{2}$, which cancels the temporal growth of the cluster size. In three-dimensional systems, a direct relation between $\chi_{4}^{\mathrm{S}}$ and the cluster size is expected only for some limited time scales corresponding to the plateau of the MSD.

\section{B. Comparison with different theories of SFD}

Among the problems of diffusion in confined geometries 7], SFD has occupied a special position which has attracted researchers from various fields of science and with a variety of mathematical approaches. Let us review some of their works briefly, with which, subsequently, the present theory will be compared.

In the oldest type of theory [93], the single-file system was modeled with an array of free Brownian particles interchanging their labels upon collision, and analyzed with a technique that makes a full use of the one-dimensional geometry, such as the Jepsen line [29]. In this line of argument, Hahn \& Kärger [25] showed that MSD for SFD can be obtained from the corresponding free-particle dynamics with the relation $\left\langle R^{2}\right\rangle \propto\left\langle\left|R_{\text {free }}\right|\right\rangle$, taking it into account that the constant of proportionality depends on the particle diameter.

Later, theories in the Fourier space emerged. The concise theory by Alexander and Pincus [23] is of this type. Generally speaking, if the spatiotemporal dynamics of the system is described by a linear equation and the system is translationally invariant, the Fourier or FourierLaplace approach is a quite natural choice. Thus, starting from the "diffusion-noise" equation, which is a linear Langevin equation for the density $\rho(x, t)$, Taloni and Lomholt [54] calculated MSD via the velocity correlation in Eq. (4.17), and Lizana et al. 36] showed that the linear dynamics is reducible to a fractional Langevin equation. These works rely on the assumption of linear dynamics, as is evident in the case of Lizana et al. [36] who denominate it the harmonization technique. We should be cautious with limitations of the harmonization, however, especially when the interparticulate potential $V(r)$ has a non-analytic point as in Eq. (A1) or in the rigid-sphere potential, as was pointed out by Ikeda et al. [71].

The simplest description based on the linear "diffusionnoise" equation cannot account for the finite-time correction which should certainly exist according to our particle-based computations. More refined linear theories may succeed in interpolating two limiting cases of $D t \ll 1 / \rho_{0}^{2}$ and $D t \gg 1 / \rho_{0}^{2}$; let us leave them aside, however, because interpolations are usually less informative than phenomenologies. Among phenomenologies on transient behavior of SFD, the theory of van Beijeren et al. 24] deserves a particular attention. This theory deals with SFD on a lattice, on the basis of the picture of migrating vacancies and making use of Eq. (4.17). The approximation of independently diffusing vacancies leads readily to the asymptotic law, $\left\langle R^{2}\right\rangle \propto \sqrt{t}$. For the cases of finite density of vacancies, van Beijeren et al. 24] noticed that a cluster of vacancies may be formed and thereby a kind of memory effect may arise. Instead of developing a systematic treatment of the memory, however, they assumed some phenomenological rules about the dynamics of a vacancy cluster and thereby calculated $\left\langle R^{2}\right\rangle$ for all $t$. We note that the picture of diffusing vacancies is both conceptually suggestive and practically useful. An asymptotic theory based on the vacancy picture can be very concise 94]. The idea of migrating defect has been used also in problems other than SFD, such as dielectric relaxation of supercooled isoamyl bromide [95]. In the context of glassy dynamics, this idea is incarnated in the kinetically constrained models, which can be regarded as a kinetic version of the free-volume theory of glasses [1, 96].

A systematic treatment of the memory effect necessitates a term with time integral. Though such a term arises in the fractional Langevin equation [36], its physical interpretation is not straightforward. Rallison 32 proposed another phenomenological theory, whose memory integral can be understood quite clearly. Suppose that $n$ Brownian particles are strongly interacting and moving together. Then it is easily shown from the Langevin equation that the effective diffusion coefficient for their center of mass is $D / n$, in the sense that

$$
\frac{\mathrm{d}}{\mathrm{d} t}\left\langle R^{2}\right\rangle=\frac{2 D}{n} \text {. }
$$

By replacing $n$ in the denominator with $\mathcal{N}(\lambda)=1+\rho_{0} \lambda$, which is the number of particles within the dynamical correlation length $\lambda=\lambda(t)$, Rallison [32] obtained

$$
\left\langle R^{2}\right\rangle=\int_{0} \frac{2 D \mathrm{~d} t}{\mathcal{N}(\lambda)}, \quad \lambda=\lambda(t)=\sqrt{4 \pi D t}
$$

Upon integration, Eq. (6.1) gives normal diffusion for small $t$, and for large $t$, it gives subdiffusion with a logarithmic correction term.

The MCT approach provides with a nonlinear theory in the form of Eq. (1.1) for $F$ and Eq. (1.5) for $F_{\mathrm{S}}$, in which the memory kernels are approximated with the products of $F$ and $F_{\mathrm{S}}$. As was mentioned in Sec. II the mathematical properties of the MCT kernels are such that they decay exponentially for the most part. This implies that SFD cannot be described by the conventional MCT. A possible approach consists in adopting Eq. (1.1) for $F$ and replacing Eq. (1.5) for $F_{\mathrm{S}}$ with another equation for tracers in which the four-point correlation is directly taken into account. The theory of Miyazaki and Yethiraj 37] for rod polymers, as well as Kollmann's theory [26], belongs to this category. We note that Kollmann [26] focused on the long-time behavior and therefore considered only the long-wave limit of Eq. (1.1), so that the nonlinear effect is ignored except for the change from $D$ to $D_{\text {c }}$.

The theories of Fedders [22] and Abel et al. 34] could be termed as a modified MCT approach, in which both Eq. (1.1) and Eq. (1.5) are essentially retained, but $M_{\mathrm{S}}$ 
is modified. Fedders [22] noticed that the summation of the diagrams must be performed with the restriction corresponding to the "no-passing" rule. In the formulation of Abel et al. 34], this restriction was taken into account by a kind of re-weighting in diagrammatic expansion. As a result, a wavenumber integral that appears in an expression related to $M_{\mathrm{S}}$ (the scaled irreducible memory function $G^{\mathrm{irr}}$ ) is changed in a delicate way. Without the "no-passing" rule, the original integral reads

$$
G^{\mathrm{irr}}(k, t) \propto \int[1-\cos (p-q)] F_{\mathrm{S}}(p, t) F(q, t) \mathrm{d} p
$$

and gives normal diffusion asymptotically. This is replaced by

$$
G_{\text {modified }}^{\text {irr }}(k, t) \propto \int F_{\mathrm{S}}(p, t) F(q, t) \mathrm{d} p
$$

due to the re-weighting, and it gives the correct anomalous diffusion.

Having reviewed main existing theories on SFD, now let us compare the present theory with them. The present theory is a nonlinear one, consisting of the Lagrangian MCT equation (4.8) and the modified Alexander-Pincus formula (2.15). The adoption of the Lagrangian description enabled us to reproduce the asymptotic law for MSD and calculate a correction to it within the liquidtheoretical framework. Some four-point space-time correlations are also calculated analytically.

One of the main differences between Eq. (4.8) for $\check{C}$ and the corresponding Eulerian MCT equation (1.1) is that the diffusing entity in Eq. (4.8) is the "free volume" between the particles, which is quite analogous to the diffusing vacancies considered by van Beijeren et al. 24 and also by other authors. While van Beijeren et al. [24] gave up a systematic treatment of the memory effects in the vacancy dynamics, the present theory treats it with a systematic approximation. The modified AlexanderPincus formula (2.15) seems to be exact in the limit of large system size. The formula (2.15) itself is linear in regard to $\check{C}$, though there is a hidden nonlinearity in the mapping from the label distance to the EulerianEuclidean distance.

The present theory gives a finite-time correction to the long-time asymptotic result, as is shown in Eq. (4.16). The correction slightly differs from that of the phenomenological equation (6.1) by Rallison [32]: probably this is attributable to the inaccuracy of $\lambda(t)$ or $\mathcal{N}(\lambda)$ assumed in Eq. (6.1). Another issue that requires further consideration is the relation between the modified Alexander-Pincus formula (2.15) and the modified MCT equation for $F_{\mathrm{S}}$, which should be understood somehow in the future.

We emphasize that there are two origins of nonlinearity, and the present MCT-based approach is capable of treating both of them in principle. One is the nonlinearity of the configurational entropy, from which the nonlinearity of $1 /(1+\psi)$ in Eq. (4.10) originates. The other is the nonlinearity whose coefficient involves $\sin \rho_{0} \sigma k$, which can be traced back to the term including $U$ in
Eq. (2.1b) and represents the effect of direct contact between the particles. Though we have omitted the analysis of the latter to limit all the results within the range of analytical calculation, it would be straightforward to deal with the cases in which these two nonlinearities are present, once a numerical scheme is constructed.

\section{Methodological insight into memory-correlation approaches}

In our derivation of Eqs. (4.8) and (4.9) for the Lagrangian correlation $\check{C}$ and the memory kernel $M$ associated with it, we took the Langevin equation for the density field as the starting point and adopted a fieldtheoretical method akin to the MSR formalism. While the derived equation itself has a form parallel to the $\mathrm{Eu}-$ lerian MCT equation (1.1), the derivation processes are quite dissimilar. Practically, Eq. (1.1) is derived directly from the microscopic equation of motion by way of the Mori-Zwanzig projection operator formalism [13 15, 97]. This is usually considered to be more convenient than the field-theoretical derivation, because the latter suffers from the difficulties due to the multiplicative noise, such as violation of the FDT. Langevin equations with multiplicative noise may draw a general criticism for the Itô-Stratonovich dilemma [53], though it can be avoided when the Onsager coefficient satisfies a certain condition [49]. Besides, in regard to the treatment of the noise correlation itself, there seems to be a subtle confusion in the literature: compare Eq. (14) in Ref. [48], Eq. (4) in Ref. 50], Eq. (2) in Ref. [51] and Eq. (6) in Ref. [54]. All these difficulties have made the Langevin equation for the density field, such as Eq. (2.2), inconvenient as a starting point.

Interestingly, when the field $\psi(\xi, t)$ is adopted instead of the conventional density field, the positions of the two methods are reversed. In contrast to the projection of the particle motion onto $\rho(\mathbf{r}, t)=\sum_{j} \delta_{j}\left(\mathbf{r}-\mathbf{r}_{j}(t)\right)$ which can be performed naturally, it is not evident how to project the motion of the particles onto $\psi=\rho_{0} / \rho-1$. Direct employment of the microscopic definition of $\rho$ for the denominator would give rise to delicate issues concerning the procedure of coarse-graining. Alternatively, a onedimensional projection-operator formalism may be possible by using $X_{j+1}-X_{j}$ as the microscopic definition of $\psi$, but this leads to another complication, because this definition of $\psi$ depends on the assumption about the ordering of the particles. On the side of the field-theoretical formalism, the difficulty of the multiplicative noise disappears quite naturally, which has allowed us to derive the Lagrangian MCT equation without violating the FDT.

Thus the combination of the Lagrangian vacancy field with the field-theoretical formalism is not less advantageous than the conventional projection operator route with the Eulerian density field. The new route that leads to the Lagrangian MCT deserves further exploration, especially if it may guide us to some improved theories of three-dimensional systems in the future. In closing the 
current section, let us discuss this possibility.

\section{Future directions: possible relevance to glassy dynamics}

We have implemented the Lagrangian description by explicitly introducing the label variable $\xi$ and thereby constructing a stretchable coordinate system that sticks to the cages everywhere. Probably some aspects of glassy dynamics, such as dynamical heterogeneity characterizable by bond breaking [16, 291, 98], may require the Lagrangian description by nature when its continuum counterpart is sought.

The Lagrangian description in higher dimensions may not be so simple as in one-dimensional cases, but it is possible. In three-dimensional cases, a triplet of label variables $(\xi, \eta, \zeta)$ is expected to be related with $\rho$ and $\mathbf{Q}$ by equations analogous to Eq. (2.5); see Eqs. (6.6) and (6.7) in Ref. 30]. Besides, we could adopt some methods from three-dimensional theories of turbulence in which Lagrangian correlations are used 60 62]. Turbulence theoreticians have even considered the Lagrangian dynamics of a tetrad (four material points) 99], whose two-time correlation involves eight points in the spacetime.

In contrast to the "Eulerian" (standard) MCT in which cage effects are represented by the memory kernel (not successful in SFD), the Lagrangian theory can dispense with the memory integral as far as the asymptotic behavior is concerned. A pivotal role is played by the modified and extended Alexander-Pincus formulae in Eqs. (2.15) and (3.2). Its linear version, namely Eq. (2.18) or its multidimensional extension, has been used in the context of glassy dynamics by several authors [18, 33], who limited themselves to the approximation with linear elasticity. Since the Eulerian and the Lagrangian variables are approximately interchangeable in the description of small elastic deformation, they did not bother to distinguish the two descriptions. Needless to say, this treatment fails when the system is more liquid-like. In an attempt to introduce the $\alpha$ relaxation into the calculation of $\chi_{4}$ based on the "elastic" theory, Toninelli et al. feared that it would make the model inconsistent, because the underlying lattice, needed to define the deformation field, would be totally melted [18]. Probably this is too pessimistic: the "melting" of the lattice does not make the theory totally inconsistent but requires more careful distinction between the Eulerian and the Lagrangian coordinates.

There will be another modification to the theory of Toninelli et al., when departing from the linear elasticity and trying to consider liquid-like behavior. Their formula corresponding to Eq. (3.2), namely Eq. (A3) in Ref. [18], reads

$$
\langle R(\tilde{\mathbf{d}}) R(\mathbf{0})\rangle \propto \int \frac{1-e^{-D \mathbf{k}^{2} t}}{\mathbf{k}^{2}} e^{-\mathrm{i} \mathbf{k} \cdot \tilde{\mathbf{d}}} \mathrm{d}^{n_{\mathrm{d}}} \mathbf{k}
$$

in our notation, as they seem to have identified the Langevin equation for the displacement field with the
Edwards-Wilkinson equation [67]. In regard to Eq. (6.4), we suspect that the vectorial character of the displacement is not adequately taken into account. Probably one needs to decompose $\mathbf{R}$ into the longitudinal and transverse components, and treat them more carefully. Unlike the two sound modes in elastic solids, the two modes in the liquids can have quite different nature: the liquid may resist compression strongly but the resistance to shear may be much weaker.

In Eq. (5.6), we have proposed to define the threedimensional $2 \mathrm{pDC}$ as a tensorial quantity $X$. Due to the isotropy and the reflectional symmetry of the system, $X$ must be a sum of the longitudinal and the transverse components:

$$
\mathrm{X}=X_{\|} \frac{\tilde{\mathbf{d}} \otimes \tilde{\mathbf{d}}}{\tilde{\mathbf{d}}^{2}}+X_{\perp}\left(\mathbb{1}-\frac{\tilde{\mathbf{d}} \otimes \tilde{\mathbf{d}}}{\tilde{\mathbf{d}}^{2}}\right) .
$$

It is quite likely that $X_{\|}$and $X_{\perp}$ will be characterized by different correlation lengths. Taking the two different correlation lengths into account, we can extend the present theory phenomenologically to the threedimensional cases. From the inferred distribution function for the displacements of two particles, shown in Appendix C, we can calculate $\chi_{4}^{\mathrm{S}}$ in the same way as in Subsec. VB, as

$$
\chi_{4}^{\mathrm{S}} \sim \frac{1}{k_{\mathrm{B}} T} \times \frac{(1-\alpha)^{2} \lambda_{\|} \lambda_{\perp}^{2}}{\left(1+\frac{2 X^{0}}{a^{2}}\right)^{3}}
$$

where $\lambda_{\|}=\lambda_{\|}(t)$ and $\lambda_{\perp}=\lambda_{\perp}(t)$ denote the two correlation lengths, $X^{0}=X^{0}(t)$ is related to the MSD of the caged particles, and $\alpha=\alpha(t)$ stands for the relative number of the particles that have hopped. Assuming the $t$-dependence of these four quantities phenomenologically as

$$
\begin{gathered}
\lambda_{\|}=\frac{\sqrt{D t}}{1+\sqrt{t / \tau}}, \quad \lambda_{\perp}=(1+\sqrt{t / \tau}) \ell_{0}, \\
X_{0}=\frac{D t}{1+D t / \ell_{0}^{2}}, \quad \alpha=\tanh \frac{t}{\tau},
\end{gathered}
$$

we can plot $\chi_{4}^{\mathrm{S}}$ against $t$ for some different values of the time scale $\tau$. The curves in Fig. 8 reproduce some basic features of the $\mathcal{Q}$-based $\chi_{4}$ calculated by Lačević et al. [86], such as the shape of the uphill that looks steeper near the peak in this semi-log plot.

To go beyond the linear theory, $n_{\mathrm{d}}$-dimensional versions of the extended Alexander-Pincus formula should be developed. We suppose that the $n_{\mathrm{d}}$-dimensional formula will contain, instead of $\check{C}$, correlations of deformation tensor. This is not so formidable as it may appear, because many components of the correlation tensor will turn out to vanish or to have the same value as some other component. The formula is now under development and will be reported elsewhere.

It is also noteworthy that, in the derivation of MCT, the difficulty of FDT violation disappeared quite naturally due to the Lagrangian description. As is pointed 


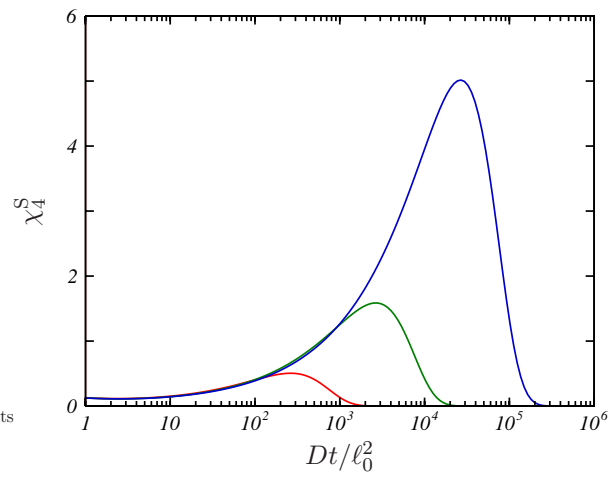

FIG. 8: (Color online) Behavior of $\chi_{4}^{\mathrm{S}}$ given by Eq. (6.5), calculated through the three-dimensional two-body displacement distribution function by phenomenologically extending the present theory to the three-dimensional cases. The three curves correspond to different values of $\tau$ : from left to right, $\tau=10^{3} \ell_{0}^{2} / D, 10^{4} \ell_{0}^{2} / D$, and $10^{5} \ell_{0}^{2} / D$.

out by Miyazaki \& Reichman [49], it has been difficult to construct realistic models which does not violate FDT and can incorporate the effect of structural changes embodied in $S(k)$ at the same time. Since the "Lagrangian MCT" is now shown to be consistent with FDT, study of its behavior for different $S(k)$ may be quite intriguing.

For possible extensions in the future, we can mention several directions. For example, one may include weak attractive interaction and analyze the effect of the change in $S(k)$ on the transient behavior of SFD. One may also study nonequilibrium behavior by driving the particles with an external force or changing the temperature suddenly. Another interesting proposal is to permit overtaking as a rare event, which may play the role of the $\alpha$ relaxation. We performed simulations with some finite interaction potential, and a preliminary result 100] shows that normal diffusion is observed for $V_{\max }=k_{\mathrm{B}} T$, while for $V_{\max }=5 k_{\mathrm{B}} T$ the behavior is essentially that of SFD in the time scale of the simulation. The problem is to make a theory that can handle the crossover between the two limiting cases. The theory allowing for rare overtaking events may bridge the gap between the purely one-dimensional SFD and the behavior of threedimensional rod polymers [32, 37].

We could also study double-file diffusion, which would be analogous to two-lane models of traffic flows. If a "lane interaction" is also introduced, the system would have also something common with the Matsukawa-Fukuyama model of friction formulated on a ladder lattice [101, 102]. The study of the double-file diffusion may shed light to many related systems in which frustration is dynamically created and annihilated, such as template-assisted pattern formation of colloid particles on a substrate with parallel channels [103], frustrated Josephson-junction arrays in a magnetic field [102, 104], and-hopefuly - also three-dimensional dense colloidal suspensions.

\section{CONCLUSION}

We have developed a nonlinear theory of SFD with a liquid-theoretical approach. The theory consists of the modified Alexander-Pincus formula (2.15) and the Lagrangian MCT equation (4.8), which gives not only the established Hahn-Kärger-Kollman law on the long-time asymptotic behavior of $\left\langle R^{2}\right\rangle$, but also a finite-time correction to it, as is shown in Eq. (4.16). Though we have focused on the nonlinearity of the configurational entropy, the proposed theoretical scheme makes it possible to deal with the other nonlinear effects in the thermodynamic potential as well, if the MCT equation is solved numerically.

Using this scheme, we have demonstrated how to calculate four-point space-time correlations, such as the $2 \mathrm{pDC}$ and $\chi_{4}^{\mathrm{S}}$. These four-point space-time correlations quantify the collective dynamics in SFD as a simple model of ideal cages involving numerous particles. The longtime behavior of $\chi_{4}^{\mathrm{S}}$ exhibits convergence to a finite value, given by $\left(\chi_{4}^{\mathrm{S}}\right)_{\text {coll }}$ in Eq. (5.17), which is an increasing function of $\rho_{0}$.

The present work, in combination with the previous one [30], is intended as several first steps toward a future theory of three-dimensional glassy liquids, which will make it possible, for example, to replace the semiphenomenological curves for $\chi_{4}^{\mathrm{S}}$ in Fig. 8 with a firstprinciple theoretical calculation. Although the present theory is still embryonic, it already suggests that one of the important ingredients of the future theory may be the displacement distribution function of two or more particles. In the case of computational analysis, probably we should not insist on some favorite statistical quantity alone, nor content ourselves with the single-particle van Hove function, but try to deduce some suitable distribution function behind the computed statistical quantities. The present analysis of one-dimensional cage dynamics and the concepts used for it will provide a useful framework both for analysis of numerical data and for new development of theory of glassy liquids.

\section{Acknowledgments}

We express our cordial gratitude to Kunimasa Miyazaki, whose lecture on SFD [39] motivated our study, and whose comments were helpful and enlightening. We also appreciate fruitful discussions with Shin-ichi Sasa, Takeshi Kawasaki, Hajime Yoshino, and So Kitsunezaki. This work was supported by Grants-in-Aid for Scientific Research (KAKEnHI) (C) No. 21540388 and (C) No. 24540404, JSPS (Japan).

\section{Appendix A: Numerical calculations}

Here we describe how we integrated the onedimensional Langevin equation (1.2), and also how we evaluated the statistical quantities, including $\left\langle R^{2}\right\rangle, D_{\mathrm{c}}$, 
and $S$, from the numerical solution. As the system contains $N$ particles in a periodic box of the size $L$, the mean density is given by $\rho_{0}=N / L$.

The potential $V$ in Eq. (1.2) was specified as

$$
V(r)= \begin{cases}V_{\max }\left(1-\frac{|r|}{\sigma}\right)^{2} & (|r| \leq \sigma) \\ 0 & (|r|>\sigma)\end{cases}
$$

with $V_{\max } \gg k_{\mathrm{B}} T$. In the present numerical calculations we adopted the value $V_{\max }=50 k_{\mathrm{B}} T$, which is high enough to forbid the overtaking of the particles completely.

The random forcing is the zero-mean Gaussian noise whose variance is given as

$$
\left\langle f_{i}(t) f_{j}\left(t^{\prime}\right)\right\rangle=2 D \delta_{i j} \delta\left(t-t^{\prime}\right)
$$

Computationally, the delta function in Eq. (A2) was discretized with the time interval $\Delta t_{f}$, and as the values of $\left(f_{0}, f_{1}, \ldots, f_{N-1}\right)$ for each time interval, $N$ independent Gaussian random numbers with the variance $2 D / \Delta t_{\mathrm{f}}$ were generated with the Mersenne twister and the BoxMuller transform.

With $V$ and $f_{i}$ given as above, in nondimensionalization of the governing equation using $\sigma, \sigma^{2} / D$, and $m$ as the units of length, time, and mass, there appears a nondimensional parameter specifiable as the ratio of $\tau_{\mathrm{B}}=m / \mu$ to the time unit $\sigma^{2} / D$, which we chose as $1: 1$. Then the time integration of Eq. (1.2) was performed with a Verlet-like scheme. The time step $\Delta t$ was taken equal to a hundredth of the time unit $\sigma^{2} / D$, and the renewal interval of the random forcing, $\Delta t_{f}$, was chosen to be the same as the time step:

$$
\Delta t=\Delta t_{\mathrm{f}}=10^{-2} \times \frac{\sigma^{2}}{D}
$$

We also tested some different choices of $\Delta t$ and $\Delta t_{\mathrm{f}}$, such as

$$
\frac{\left(\Delta t, \Delta t_{\mathrm{f}}\right)}{\sigma^{2} / D}=\left(10^{-3}, 10^{-3}\right) \quad \text { or } \quad\left(10^{-3}, 10^{-2}\right)
$$

To bring the system into equilibrium, we started each calculation at $t=-\mathcal{T}_{\mathrm{w}}$, introducing a sufficiently long waiting time $\mathcal{T}_{\mathrm{w}}$ (typically $\mathcal{T}_{\mathrm{w}}=10^{4} \sigma^{2} / D$ ), and waited till $t=0$. Then, from the simulation data recorded for $0 \leq t \leq t_{\max }$, we calculated a desired statistical quantity as the average for $n$ samples extracted from the data by a time shift. For example, $\left\langle R^{2}\right\rangle$ is calculated as

$$
\left\langle R\left(t^{\prime}\right)^{2}\right\rangle=\frac{1}{n N} \sum_{l=0}^{n-1} \sum_{i=0}^{N-1}\left[X_{i}\left(t_{l}+t^{\prime}\right)-X_{i}\left(t_{l}\right)\right]^{2}
$$

where $t_{l}$ denotes the starting time of the $l$-th sample. Care must be taken so that the maximal value of $t^{\prime}$ in Eq. (A4), which equals the span of each sample, should not exceed the waiting time $\mathcal{T}_{\mathrm{w}}$; a result for a longer span will expose insufficiency of equilibration. Typically
TABLE I: Numerical values of $S(0)$ and $D_{\text {c }} / D$ computed for three different values of the density.

\begin{tabular}{lccc}
\hline$\rho_{0}$ & $(1 / 4) \sigma^{-1}$ & $(1 / 8) \sigma^{-1}$ & $(1 / 16) \sigma^{-1}$ \\
\hline $1-2 \rho_{0} \sigma$ & 0.500 & 0.750 & 0.875 \\
$S(0)$ & 0.624 & 0.787 & 0.888 \\
$D_{\mathrm{c}} / D$ & 1.59 & 1.27 & 1.12 \\
$S(0) \sqrt{D_{\mathrm{c}} / D}$ & 0.79 & 0.89 & 0.94 \\
\hline
\end{tabular}

we chose $t_{\text {max }}=5 \mathcal{T}_{\mathrm{w}}, n=100$, and $t_{l}=l\left(t_{\max }-\mathcal{T}_{\mathrm{w}}\right) / n$, allowing the samples to overlap.

The computation of $X_{R}(\tilde{d}, t)$, shown in Fig. 6] was performed with discretization of Eq. (5.2) in which the delta function was approximated by a statistical bin $0.5 \sigma$ in width. After recording $X_{i}\left(t_{l}\right)$ for every particle, we classified every pair $(i, j)$ into a statistical bin according to the "initial" distance $X_{j}\left(t_{l}\right)-X_{i}\left(t_{l}\right)$, so that the $\kappa$-th bin contains the pairs for which $\left|X_{j}\left(t_{l}\right)-X_{i}\left(t_{l}\right)-\tilde{d}_{\kappa}\right|$ is smaller than the half width of the bin. Then we calculated $X_{R}\left(\tilde{d}_{\kappa}, t\right)$ as the average of $R_{i}(t) R_{j}(t)$ for the $\kappa$-th bin, where $R_{i}(t)=X_{i}\left(t_{l}+t\right)-X_{i}\left(t_{l}\right)$. If, instead, the sum of the absolute values of the data in each bin were calculated, this would be analogous to the quantity studied by Donati et al. 44].

The collective diffusion coefficient $D_{\mathrm{c}}$ is determined from the temporal decay of the dynamical structure factor $F(q, t)$ [35]. We computed $F(q, t)$ for $0<q \ll \rho_{0}$ and made a linear fit for $\log F(q, t)=\log S(q)-D_{\mathrm{c}} q^{2} t$ to obtain the values of $S(q)$ and $D_{\text {c }}$ as the fitting parameters. After taking an average for several small values of $q$, the results are summarized in Table \. These values are used in evaluation of the theoretical predictions, such as Eq. (3.3), and also for rescaling of the horizontal axis in Fig. 5 .

\section{Appendix B: Direct-interaction approximation}

The main idea of DIA [77, 78] for evaluation of the triple correlation

$$
\langle\check{\psi}(-p, t) \check{\psi}(-q, t) \check{\psi}(-k, 0)\rangle=\langle\check{\psi}(p, t) \check{\psi}(q, t) \check{\psi}(k, 0)\rangle^{*}
$$

with the asterisk denoting the complex conjugate, is to utilize the property of $\mathcal{V}=\left(\mathcal{V}_{\alpha}^{\beta \gamma}\right)$ that, out of its $N^{3}$ components, almost all are zero because the condition $\alpha+\beta+\gamma=0$ is not satisfied. The nonzero components of $\mathcal{V}$ constitute what we call triad interactions: rewriting Eq. (2.12) as

$$
\left(\partial_{t}+\mu_{\alpha}\right) \check{\psi}(\alpha, t)=\sum_{\beta, \gamma} \mathcal{V}_{\alpha}^{\beta \gamma} \check{\psi}^{*}(\beta, t) \check{\psi}^{*}(\gamma, t)+\rho_{0} \check{f}_{\mathrm{L}}(\alpha, t)
$$

makes it clearer that $\mathcal{V}_{\alpha}^{\beta \gamma}$ engages in connecting the "triad" that consists of $\alpha, \beta$, and $\gamma$. If we visualize each triad interaction as a triangle on a graph, the property of $\mathcal{V}$ is such that no triangle shares its side with other triangles. Therefore, if we "switch off" a single triad, say, $\{p, q, k\}$ (with which we mean $\mathcal{V}_{k}^{p q}, \mathcal{V}_{k}^{q p}, \mathcal{V}_{p}^{q k}, \mathcal{V}_{p}^{k q}$, 
$\mathcal{V}_{q}^{k p}$, and $\mathcal{V}_{q}^{p k}$; note the symmetry in regard to the interchange of the superscripts, $\mathcal{V}_{k}^{p q}=\mathcal{V}_{k}^{q p}$ etc.), the direct interactions between the three modes $p, q, k$ are lost.

To concretize this idea, let us suppose that an artificial forcing term

$$
I_{\alpha}=-\theta\left(t-t_{0}\right) \times \begin{cases}2 \mathcal{V}_{k}^{p q} \check{\psi}^{*}(p, t) \check{\psi}^{*}(q, t) & (\alpha=k) \\ 2 \mathcal{V}_{p}^{q k} \check{\psi}^{*}(q, t) \check{\psi}^{*}(k, t) & (\alpha=p) \\ 2 \mathcal{V}_{q}^{k p} \check{\psi}^{*}(k, t) \check{\psi}^{*}(p, t) & (\alpha=q) \\ 0 & \text { (otherwise) }\end{cases}
$$

designed to cancel a single triad $\{p, q, k\}$, is applied to the system given by Eq. (2.12]). We denote the solution to this artificial system with $\breve{\psi}_{0}=\breve{\psi}_{0}(\{p, q, k\} ; \alpha, t)$. One of the two main assumptions of DIA is that the three selected modes, namely $\breve{\psi}_{0}(p, t), \check{\psi}_{0}(q, t)$, and $\check{\psi}_{0}(k, t)$ in this case, become uncorrelated, since the forcing $I$ cancels the direct interactions. On the other hand, $I$ is regarded as a small perturbation, because it cancels only a single triad interaction and there remain still a large number of triads connecting, say, $p$ and $q$ indirectly. Therefore the difference $\check{\psi}_{1}=\breve{\psi}-\breve{\psi}_{0}$ is assumed to be small, which is the second main assumption of DIA.

Due to these assumptions, the triple correlation is expanded as

$$
\begin{aligned}
\langle\check{\psi}(p, t) \check{\psi}(q, t) \check{\psi}(k, 0)\rangle= & \left\langle\check{\psi}_{0}(p, t) \check{\psi}_{0}(q, t) \check{\psi}_{0}(k, 0)\right\rangle \\
& +\left\langle\check{\psi}_{1}(p, t) \check{\psi}_{0}(q, t) \check{\psi}_{0}(k, 0)\right\rangle \\
& +\left\langle\check{\psi}_{0}(p, t) \check{\psi}_{1}(q, t) \check{\psi}_{0}(k, 0)\right\rangle \\
& +\left\langle\check{\psi}_{0}(p, t) \check{\psi}_{0}(q, t) \check{\psi}_{1}(k, 0)\right\rangle \\
& +O\left(\check{\psi}_{1}^{2}\right),
\end{aligned}
$$

and the zero-th term, $\left\langle\check{\psi}_{0}(p, t) \check{\psi}_{0}(q, t) \check{\psi}_{0}(k, 0)\right\rangle$, vanishes. Since $I$ is a small perturbation and $\psi_{1}$ is a response to it, formally $\psi_{1}$ can be expressed in terms of the propagator $G$ as

$$
\check{\psi}_{1}(\alpha, t)=-\int_{t_{0}}^{t} \mathrm{~d} t^{\prime} \sum_{\alpha^{\prime}} G\left(\alpha, t ; \alpha^{\prime}, t^{\prime}\right) I_{\alpha^{\prime}}\left(t^{\prime}\right)
$$

for $t>t_{0}$. We substitute Eq. (B2) into each term on the right-hand side of Eq. (B1) to find, to our surprise, that the result is naturally factorized due to the assumption of DIA that $\check{\psi}_{0}(p, t), \check{\psi}_{0}(q, t)$, and $\check{\psi}_{0}(k, t)$ are uncorrelated.

By applying the decomposition $\check{\psi}=\check{\psi}_{0}+\check{\psi}_{1}$ to each triple correlation term in the equation for $\partial_{t} \check{C}$, we are led to Eq. (4.1). Similarly, the equation for $\partial_{t} \bar{G}$ contains $\left\langle\breve{\psi}_{0} G\right\rangle$, which is evaluated with the aid of the DIA decomposition of $G$, resulting in Eq. (4.2). For more details, see Refs. [78, 79].

\section{Appendix C: Three-dimensional calculation of $\chi_{4}^{\mathrm{S}}$}

Here we outline how to calculate $\chi_{4}^{\mathrm{S}}$ by phenomenologically extending the present theory to the threedimensional cases. The displacement correlation tensor
$\mathrm{X}$ is related to the distribution function $P\left(\tilde{\mathbf{d}} ; \mathbf{R}_{i}, \mathbf{R}_{j}\right)$ by

$$
\mathrm{X}(\tilde{\mathbf{d}})=\iint \mathbf{R}_{i} \otimes \mathbf{R}_{j} P\left(\tilde{\mathbf{d}} ; \mathbf{R}_{i}, \mathbf{R}_{j}\right) \mathrm{d}^{3} \mathbf{R}_{i} \mathrm{~d}^{3} \mathbf{R}_{j}
$$

in what follows, the $\tilde{\mathbf{d}}$-dependence of $P$ is taken for granted and therefore omitted. The relation can be inverted if the functional form of $P$ is known. In particular, if a multivariate Gaussian distribution (which we denote with $\left.P_{0}\right)$ is assumed, $P=P_{0}$ can be factorized as

$$
P_{0}\left(\mathbf{R}_{i}, \mathbf{R}_{j}\right)=P_{\|}\left(\mathbf{R}_{i}^{\|}, \mathbf{R}_{j}^{\|}\right) P_{\perp}\left(\mathbf{R}_{i}^{\perp}, \mathbf{R}_{j}^{\perp}\right)
$$

by splitting $\mathbf{R}$ into the longitudinal and transverse components as $\mathbf{R}=\mathbf{R}^{\|}+\mathbf{R}^{\perp}$ (so that $\mathbf{R}^{\|} \| \tilde{\mathbf{d}}$ and $\mathbf{R}^{\perp} \perp \tilde{\mathbf{d}}$ ). Then we introduce $X^{0}$ such that

$$
X_{\|}(\tilde{d}=0)=X_{\perp}(\tilde{d}=0)=X^{0}
$$

and write the two factors explicitly as

$$
\begin{aligned}
P_{\|}= & \frac{1}{2 \pi \sqrt{\Delta_{\|}}} \exp \left[-\frac{X^{0}\left(R_{i}^{\|^{2}}+R_{j}^{\|^{2}}\right)-2 X_{\|}(\tilde{d}) R_{i}^{\|} R_{j}^{\|}}{2 \Delta_{\|}}\right], \\
P_{\perp}= & \frac{1}{(2 \pi)^{2} \Delta_{\perp}} \times \\
& \quad \exp \left[-\frac{X^{0}\left(\mathbf{R}_{i}^{\perp^{2}}+\mathbf{R}_{j}^{\perp^{2}}\right)-2 X_{\perp}(\tilde{d}) \mathbf{R}_{i}^{\perp} \cdot \mathbf{R}_{j}^{\perp}}{2 \Delta_{\perp}}\right],
\end{aligned}
$$

where $\Delta_{\|}=\left(X^{0}\right)^{2}-X_{\|}(\tilde{d})^{2}$ etc.

Then the calculation of $\chi_{4}^{\mathrm{S}}$ will be carried out in a way analogous to the $1 \mathrm{D}$ cases. Subsequently, assuming that two correlation lengths, $\lambda_{\|}=\lambda_{\|}(t)$ and $\lambda_{\perp}=\lambda_{\perp}(t)$, can be introduced so that

$$
\begin{aligned}
& X_{\|} \sim X^{0}(t) \Phi_{\|}\left(\tilde{d} / \lambda_{\|}(t)\right), \\
& X_{\perp} \sim X^{0}(t) \Phi_{\perp}\left(\tilde{d} / \lambda_{\perp}(t)\right),
\end{aligned}
$$

we estimate the number of the particles contributing to the sum as $N_{\text {coll }} \sim \rho_{0} \lambda_{\|} \lambda_{\perp}^{2}$, which leads to

$$
\chi_{4}^{\mathrm{S}} \sim \frac{1}{k_{\mathrm{B}} T} \times \frac{\lambda_{\|} \lambda_{\perp}^{2}}{\left(1+\frac{2 X^{0}}{a^{2}}\right)^{3}}
$$

as a three-dimensional counterpart of Eq. (5.16a).

Of course, Eq. (C2) needs to be modified by taking $\alpha$ relaxation into account. We introduce $\alpha=\alpha(t)$ denoting the relative number of the particles that have hopped by the time $t$, and assume the distribution function in the form

$$
\begin{aligned}
P & \left(\mathbf{R}_{i}, \mathbf{R}_{j}\right) \\
& = \begin{cases}(1-\alpha)^{2} P_{0}+2 \alpha(1-\alpha) P_{1}+\alpha^{2} P_{2} & (i \neq j) \\
(1-\alpha) P_{0}+\alpha P_{2} & (i=j),\end{cases}
\end{aligned}
$$


where $P_{0}$, governing the pairs of caged particles, is given by Eq. (C1). Note that, by integrating $P\left(\mathbf{R}_{i}, \mathbf{R}_{j}\right)$ in regard to the second argument $\mathbf{R}_{j}$, Eq. (C3) reduces to the van Hove function in the form

$$
P(\mathbf{R})=(1-\alpha) P_{\text {cage }}(\mathbf{R})+\alpha P_{\text {hop }}(\mathbf{R}) .
$$

This implies that $\alpha=\alpha(t)$ can be determined, in principle, as a fitting parameter for the van Hove function. If we assume, for simplicity, that the correlation of dis- placements is totally lost after the hopping, we have

$$
\begin{aligned}
& P_{1}\left(\mathbf{R}_{i}, \mathbf{R}_{j}\right)=\frac{P_{\text {hop }}\left(\mathbf{R}_{i}\right) P_{\text {cage }}\left(\mathbf{R}_{j}\right)+P_{\text {cage }}\left(\mathbf{R}_{i}\right) P_{\text {hop }}\left(\mathbf{R}_{j}\right)}{2}, \\
& P_{2}\left(\mathbf{R}_{i}, \mathbf{R}_{j}\right)=P_{\text {hop }}\left(\mathbf{R}_{i}\right) P_{\text {hop }}\left(\mathbf{R}_{j}\right) .
\end{aligned}
$$

Using the distribution function in Eq. (C3) supplemented with the above expressions, we evaluate $\chi_{4}^{\mathrm{S}}$ and obtain Eq. (6.5).
[1] T. M. Squires and S. R. Quake, Rev. Mod. Phys. 77, 977 (2005).

[2] M. S. Hale and J. G. Mitchell, Nano Letters 1, 617 (2001).

[3] A. Kusumi, Y. Sako, and M. Yamamoto, Biophysical Journal 65 (1993).

[4] B. Alberts, A. Johnson, J. Lewis, M. Raff, K. Roberts, and P. Walter, Molecular Biology of the Cell (Garland Science, New York, 2007), 5th ed., ISBN 9780815341055 .

[5] K. Sekimoto, Stochastic energetics (Springer-Verlag, 2010).

[6] P. Hänggi and F. Marchesoni, Rev. Mod. Phys. 81, 387 (2009).

[7] P. S. Burada, P. Hänggi, F. Marchesoni, G. Schmid, and P. Talkner, ChemPhysChem 10, 45 (2009).

[8] R. Zwanzig, J. Phys. Chem. 96, 3926 (1992).

[9] D. Reguera, G. Schmid, P. S. Burada, J. M. Rubí, P. Reimann, and P. Hänggi, Phys. Rev. Lett. 96, 130603 (2006).

[10] D. Reguera, A. Luque, P. S. Burada, G. Schmid, J. M. Rubí, and P. Hänggi, Phys. Rev. Lett. 108, 020604 (2012).

[11] L. Berthier and G. Biroli, Rev. Mod. Phys. 83, 587 (2011).

[12] A. J. Liu and S. R. Nagel, Jamming and Rheology: Constrained Dynamics on Microscopic and Macroscopic Scales (Taylor \& Francis, London, 2001).

[13] W. Götze and L. Sjögren, Rep. Prog. Phys. 55, 241 (1992).

[14] W. Götze, Complex Dynamics of Glass-Forming Liquids: A mode-coupling theory (Oxford University Press, New York, 2009), ISBN 978-0-19-923534-6.

[15] D. R. Reichman and P. Charbonneau, J. Stat. Mech. p. P05013 (2005).

[16] R. Yamamoto and A. Onuki, Phys. Rev. E 58, 3515 (1998).

[17] L. Berthier, G. Biroli, J.-P. Bouchaud, L. Cipelletti, and W. van Saarloos, eds., Dynamical Heterogeneities in Glasses, Colloids, and Granular Media (Oxford University Press, Oxford, 2011).

[18] C. Toninelli, M. Wyart, L. Berthier, G. Biroli, and J.-P. Bouchaud, Phys. Rev. E 71, 041505 (2005).

[19] G. Biroli, J.-P. Bouchaud, K. Miyazaki, and D. R. Reichman, Phys. Rev. Lett. 97, 195701 (2006).

[20] T. E. Harris, J. Appl. Probab. 2, 323 (1965).

[21] D. W. Jepsen, J. Math. Phys. 6, 405 (1965).

[22] P. A. Fedders, Phys. Rev. B 17, 40 (1978).

[23] S. Alexander and P. Pincus, Phys. Rev. B 18, 2011 (1978).
[24] H. van Beijeren, K. W. Kehr, and R. Kutner, Phys. Rev. B 28, 5711 (1983).

[25] K. Hahn and J. Kärger, J. Phys. A: Math. Gen. 28, 3061 (1995).

[26] M. Kollmann, Phys. Rev. Lett. 90, 180602 (2003).

[27] A. Taloni and F. Marchesoni, Phys. Rev. Lett. 96, 020601 (2006).

[28] E. Barkai and R. Silbey, Phys. Rev. Lett. 102, 050602 (2009).

[29] E. Barkai and R. Silbey, Phys. Rev. E 81, 041129 (2010).

[30] Ooshida Takeshi, S. Goto, T. Matsumoto, A. Nakahara, and M. Otsuki, J. Phys. Soc. Japan 80, 074007 (2011).

[31] J.-B. Delfau, C. Coste, and M. Saint Jean, Phys. Rev. E 85, 061111 (2012).

[32] J. M. Rallison, J. Fluid Mech. 186, 471 (1988).

[33] A. Lefèvre, L. Berthier, and R. Stinchcombe, Phys. Rev. E 72, 010301(R) (2005).

[34] S. M. Abel, Y.-L. S. Tse, and H. C. Andersen, Proc. Natl. Acad. Sci. USA 106, 15142 (2009).

[35] C. Lutz, M. Kollmann, and C. Bechinger, Phys. Rev. Lett. 93, 026001 (2004).

[36] L. Lizana, T. Ambjörnsson, A. Taloni, E. Barkai, and M. A. Lomholt, Phys. Rev. E 81, 051118 (2010).

[37] K. Miyazaki and A. Yethiraj, J. Chem. Phys. 117, 10448 (2002).

[38] K. Miyazaki, Bussei Kenkyû 88, 621 (2007), [in Japanese].

[39] K. Miyazaki, in Meeting Absrtacts of the Physical Society of Japan (2008 Annual Meeting) Part 2 (2008), p. 393, 26pWE-11 [in Japanese].

[40] L. D. Landau and E. M. Lifshitz, Fluid Mechanics, vol. 6 of Theoretical Physics (Butterworth-Heinemann, Oxford, 1987).

[41] A. Bennett, Lagrangian fluid dynamics (Cambridge University Press, Cambridge, 2006), ISBN 0-521-853109.

[42] T. Muranaka and Y. Hiwatari, Phys. Rev. E 51, R2735 (1995).

[43] Y. Hiwatari and T. Muranaka, J. Non-Cryst. Solids 235-237, 19 (1998).

[44] C. Donati, S. C. Glotzer, and P. H. Poole, Phys. Rev. Lett. 82, 5064 (1999).

[45] B. Doliwa and A. Heuer, Phys. Rev. E 61, 6898 (2000).

[46] A. Taloni, A. Chechkin, and J. Klafter, Phys. Rev. E 82, 061104 (2010).

[47] A. Taloni, A. Chechkin, and J. Klafter, Europhys. Lett. 97, 30001 (2012).

[48] D. S. Dean, J. Phys. A: Math. Gen. 29, L613 (1996).

[49] K. Miyazaki and D. R. Reichman, J. Phys. A: Math. 
Gen. 38, L343 (2005).

[50] A. Andreanov, G. Biroli, and A. Lefèvre, J. Stat. Mech. p. P07008 (2006).

[51] B. Kim and K. Kawasaki, J. Phys. A: Math. Theor. 40, F33 (2007).

[52] B. Kim and K. Kawasaki, J. Stat. Mech. p. P02004 (2008).

[53] N. G. van Kampen, Stochastic processes in physics and chemistry (Elsevier, 2007), 3rd ed.

[54] A. Taloni and M. A. Lomholt, Phys. Rev. E 78, 051116 (2008).

[55] When comparing Eq. (2.3) with Eq. (6) in Ref. [54], care should be taken of the difference in the definition of the random force: $\partial_{x}$ is included in our definition of $f_{\rho}$, which corresponds to the divergence of the noise term in Ref. [54].

[56] K. Kawasaki, Physica A 208, 36 (1994).

[57] K. Kawasaki, Journal of Statistical Physics 93, 527 (1998).

[58] V. Krakoviack, Phys. Rev. E 79, 061501 (2009).

[59] S. K. Schnyder, F. Höfling, T. Franosch, and T. Voigtmann, Journal of Physics: Consensed Matters 23, 234121 (2011).

[60] R. H. Kraichnan, Physics of Fluids 8, 575 (1965).

[61] Y. Kaneda, J. Fluid Mech. 107, 131 (1981).

[62] S. Kida and S. Goto, J. Fluid Mech. 345, 307 (1997).

[63] M. Spivak, Calculus on Manifolds: A Modern Approach to Classical Theorems of Advanced Calculus (W.A. Benjamin Inc., New York, 1965), ISBN 0-8053-9021-9.

[64] It is sometimes more convenient to regard the triad summation, such as the one in Eq. (2.12), simply as $\sum_{p} \sum_{q}$ with $\mathcal{V}_{k}^{p q}$ given by Eq. 2.13) if the triad condition is satisfied and otherwise $\mathcal{V}_{k}^{p q}=0$.

[65] Terms containing $\delta_{k, k^{\prime} \pm N}, \delta_{k, k^{\prime} \pm 2 N}, \ldots$ are omitted from Eq. (2.14), as they are not important for the behavior of the long-wave modes.

[66] J.-B. Delfau, C. Coste, and M. Saint Jean, Phys. Rev. E 84, 011101 (2011).

[67] S. F. Edwards and D. R. Wilkinson, Proc. R. Soc. London, Ser. A 381, 17 (1982).

[68] M. Doi and S. F. Edwards, The Theory of Polymer Dynamics (Oxford, 1986).

[69] The factor $L^{-2}$ is included in the definition of $\breve{C}$ so that $\check{C}$ has the dimension of the square of the density. It is a matter of choice whether to include it or not.

[70] K. Honda, Phys. Rev. E 55, R1235 (1997).

[71] A. Ikeda, L. Berthier, and G. Biroli, J. Chem. Phys. 138, 12A507 (2013).

[72] J. Krug, Adv. in Phys. 46, 139 (1997).

[73] S. P. Das and G. F. Mazenko, Phys. Rev. A 34 (1986).

[74] R. Schmitz, J. W. Dufty, and P. De, Phys. Rev. Lett. 71, 2066 (1993).

[75] P. C. Martin, E. D. Siggia, and H. A. Rose, Phys. Rev. A 8, 423 (1973).
[76] T. H. Nishino and H. Hayakawa, Phys. Rev. E 78, 061502 (2008).

[77] R. H. Kraichnan, J. Fluid Mech. 5, 497 (1959).

[78] S. Goto and S. Kida, Physica D 117, 191 (1998).

[79] Ooshida Takeshi, S. Goto, T. Matsumoto, A. Nakahara, and M. Otsuki, Progress of Theoretical Physics Supplement 195, 157 (2012).

[80] U. M. B. Marconi, A. Puglisi, L. Rondoni, and A. Vulpiani, Physics Reports 461, 111 (2008).

[81] H. Risken, The Fokker-Planck equation: methods of solution and applications (Springer, 1996), 2nd ed.

[82] In the case of Navier-Stokes turbulence, the selfinteraction is assured to vanish due to the incompressibility condition.

[83] V. Krakoviack, Phys. Rev. E 75, 031503 (2007).

[84] J. K. Percus, Phys. Rev. A 9, 557 (1974).

[85] S. C. Glotzer, V. N. Novikov, and T. B. Schrøder, J. Chem. Phys. 112, 509 (2000).

[86] N. Lačević, F. W. Starr, T. B. Schrøder, and S. C. Glotzer, J. Chem. Phys. 119, 7372 (2003).

[87] L. Berthier, Phys. Rev. E 69, 020201(R) (2004).

[88] C. Dasgupta, A. V. Indrani, S. Ramaswamy, and M. K. Phani, Europhys. Lett. 15, 307 (1991).

[89] O. Dauchot, G. Marty, and G. Biroli, Phys. Rev. Lett. 95, $265701(2005)$.

[90] F. Lechenault, O. Dauchot, G. Biroli, and J. P. Bouchaud, Europhys. Lett. 83, 46002 (2008).

[91] H. Shiba, T. Kawasaki, and A. Onuki, arXiv:1205.6090.

[92] Though it may be more appropriate to denote the $\mathcal{Q}_{\mathrm{S}^{-}}$ based $\chi_{4}$ with $\chi_{4}^{\mathcal{Q}_{\mathrm{S}}}$ following the notation of Dauchot et al. [89], here we have chosen to write $\chi_{4}^{\mathrm{S}}$. This is actually a somewhat arbitrary choice, which we made simply because it is more friendly to the eyes.

[93] D. G. Levitt, Phys. Rev. A 8, 3050 (1973).

[94] J. Kärger, Phys. Rev. A 45, 4173 (1992).

[95] S. H. Glarum, J. Chem. Phys. 33, 639 (1960).

[96] M. Sellitto and J. J. Arenzon, Phys. Rev. E 62, 7793 (2000).

[97] H. Mori, Prog. Theor. Phys. 33, 423 (1965).

[98] T. Kawasaki and A. Onuki, arXiv:1210.0369v2.

[99] M. Chertkov, A. Pumir, and B. I. Shraiman, Physics of Fluids 11, 2394 (1999).

[100] Ooshida Takeshi, M. Otsuki, S. Goto, A. Nakahara, and T. Matsumoto, Nagare 31 (2012), [in Japanese].

[101] H. Matsukawa and H. Fukuyama, Phys. Rev. B 49, 17286 (1994).

[102] H. Yoshino, T. Nogawa, and B. Kim, Progress of Theoretical Physics Supplement 184, 153 (2010).

[103] C. Mondal and S. Sengupta, Phys. Rev. E 85, 020402(R) (2012).

[104] H. Yoshino, T. Nogawa, and B. Kim, Phys. Rev. Lett. 105, 257004 (2010). 